Draft VERsion MAY 28, 2022

Typeset using $\mathrm{LAT}_{\mathrm{E}} \mathrm{X}$ preprint2 style in AASTeX61

\title{
CCD PARALLAXES FOR 309 LATE-TYPE DWARFS AND SUBDWARFS
}

\author{
Conard C. Dahn,${ }^{1}$ Hugh C. Harris,${ }^{1}$ John P. Subasavage,${ }^{1}$ Harold D. Ables,${ }^{1}$ \\ Blaise J. Canzian, ${ }^{2}$ Harry H. Guetter, ${ }^{1}$ Fred H. Harris,${ }^{1}$ Arne H. Henden, ${ }^{3}$ S. K. Leggett,${ }^{4}$ \\ Stephen E. Levine, ${ }^{5}$ Christian B. Luginbuhl, ${ }^{1}$ Alice B. Monet,${ }^{1}$ David G. Monet,${ }^{1}$ \\ Jeffrey A. Munn, ${ }^{1}$ Jeffrey R. Pier, ${ }^{1}$ Ronald C. Stone, ${ }^{1}, *$ Frederick J. Vrba, ${ }^{1}$ \\ Richard L. Walker, ${ }^{1, *}$ and Trudy M. Tilleman ${ }^{1}$ \\ ${ }^{1}$ US Naval Observatory, Flagstaff Station, 10391 W. Naval Observatory Road, Flagstaff, AZ 86005-8521, USA \\ ${ }^{2}$ L-3 Communications/Brashear, 615 Epsilon Dr., Pittsburgh, PA 15238-2807, USA \\ ${ }^{3}$ AAVSO, Cambridge, MA 02138, USA \\ ${ }^{4}$ Gemini Observatory, Northern Operations Center, 670 N. A'ohoku Place, Hilo, HI 96720, USA \\ ${ }^{5}$ Lowell Observatory, 1400 W. Mars Hill Road, Flagstaff, AZ 86001-4499, USA
}

\section{ABSTRACT}

New, updated, and/or revised CCD parallaxes determined with the Strand Astrometric Reflector at the Naval Observatory Flagstaff Station (NOFS) are presented. Included are results for 309 latetype dwarf and subdwarf stars observed over the 30+ years that the program operated. For 124 of the stars, parallax determinations from other investigators have already appeared in the literature and we compare the different results. Also included here is new or updated VI photometry on the Johnson-Kron-Cousins system for all but a few of the faintest targets. Together with 2MASS $J H K_{s}$ near-infrared photometry, a sample of absolute magnitude versus color and color versus color diagrams are constructed. Since large proper motion was a prime criterion for targeting the stars, the majority turn out to be either M-type subdwarfs or late M-type dwarfs. The sample also includes 50 dwarf or subdwarf L-type stars, and four T dwarfs. Possible halo subdwarfs are identified in the sample based on tangential velocity, subluminosity, and spectral type. Residuals from the solutions for parallax and proper motion for several stars show evidence of astrometric perturbations.

Keywords: astrometry, parallaxes, proper motions, stars: distances, stars: late-type, subdwarfs

Corresponding author: Subasavage, J. P.

jsubasavage@nofs.navy.mil

* Deceased 


\section{INTRODUCTION}

- Ground-based trigonometric parallax observations have provided the anchor for the cosmological distance ladder for over a century. Such parallax determinations were primarily made photographically using classical refracting telescopes with their associated accidental and systematic errors. van Altena et al. (1995) presented a compilation (hereafter referred to as YPC4) of all trigonometric stellar parallaxes available through 1995 November - 15,994 determinations for a total of 8112 individual stars. Included is a thorough analysis of the associated errors for each telescope employed. During the late 1980s improved precisions were realized by using hypersensitized high-DQE photographic emulsions and more sophisticated plate measuring machines. However, parallax determinations with formal mean errors below $\sim 2$ mas were rarely obtained photographically.

The U.S. Naval Observatory 61-in Astrometric Reflector (Strand 1964) was commissioned at the Flagstaff Station in late 1963 and commenced regular observations for stellar trigonometric parallaxes in mid 1964. The Strand Astrometric Reflector was designed specifically for high-precision narrow-field differential astrometric observations. Optically, it consists of a parabolic primary mirror along with a flat secondary mirror and arranged in a Cassegrain configuration. With no optical power from the secondary mirror, the main optical aberration is coma from the primary mirror. Only in exceptionally good seeing was the coma detectable within the field generally employed for photographic parallax observations on $5 \times 7$-inch Kodak plates. Furthermore, since the focal plane is flat and the distortion produced by coma is radially symmetric around the optical axis, it is allowed for by the scale terms in the linear plate constant solutions employed. On the negative side, such an optical configuration necessitates a large secondary mirror (with cell, approxi- mately 40 inch diameter) in order to produce an unvignetted field of $\sim 30$ arcmin in radius. This effectively reduces the light-gathering power of the telescope to one of $\sim 49$ inch clear aperture. The effective focal plane scale of the Strand Reflector has been monitored both with time and position across the FOV employed. To four significant digits, it is constant at 13.55 arcsec $\mathrm{mm}^{-1}$.

Operating in photographic mode through mid 1994, a total of 1013 Naval Observatory Flagstaff Station (NOFS) parallaxes were derived. While parallax determinations with uncertainties in the $2-4$ mas range were routinely obtained, precisions in the $1-2$ mas range were still rarely realized. Speculation grew that this formal error "barrier" might be either due to either fundamentals associated with making ground-based observations through the Earth's turbulent atmosphere (Lindegren 1980; Gatewood 1987; Han 1989) and/or due to slight emulsion shifts perhaps associated with plate processing (Levinson \& Ianna 1977). However, when the NOFS parallax program transitioned from photography to a CCD detector in the mid 1980 s, it soon became apparent that parallax uncertainties at the $0.5-1.2$ mas level could routinely be realized.

In this paper, we present a compilation of NOFS CCD parallaxes for all 309 of the latetype dwarf and subdwarf stars that have been observed with the Strand Astrometric Telescope. The results presented here include all observations obtained through 2016 June and employ all of the processing updates outlined in the following section. A similar compilation for $\sim 170$ white dwarf stars will be presented in a separate paper, which is in preparation. Not included in this paper are the results for the three perturbation binaries for which preliminary results were presented in Harris et al. (2015) and which are under continuing observation. NOFS results for the perturbation binary LSR1610-00 
have recently been presented by Koren et al. (2016) and will not be repeated here. Also not included here are results for 10 dwarf carbon stars being examined for astrometric perturbations with continuing observations.

Section 2 summarizes the evolution of the NOFS CCD parallax work, including various refinements and improvements - both in instrumentation and data processing - which have been instituted over the 25 year duration of the program. The final astrometric results are presented in Section 3 and the supporting photometric results for the target stars are given in Section 4. Section 5 presents several color-magnitude and color-color diagrams constructed from our database. An additional discussion regarding anticipated Gaia (Gaia Collaboration et al. 2016a,b) contributions for latetype dwarfs and subdwarfs follows in Section 6 .

\section{EVOLUTION OF THE NOFS CCD PARALLAX PROGRAM}

By way of background, we note that the NOFS CCD parallax program evolved from the efforts initiated by $D$. Monet using the KPNO 4m/CCD instrumentation (Monet \& Dahn 1983). The history of and details on the early USNO efforts can be found in Monet et al. (1992) where results are presented for 72 stars. The TI800 camera employed in this early work was very crude by modern standards in that the chip was cosmetically poor, having several blocked columns, and, at the Strand Telescope, provided a limited useful FOV of only $2.5 \times 2.5$ arcminutes. On the positive side, the small pixel size $\left(0.206^{\prime \prime}\right.$ on the sky) provided excellent image sampling, even in the very best seeing conditions realized at the Strand Telescope $\left(\sim 0.5^{\prime \prime} \mathrm{FWHM}\right)$.

As noted in Monet et al. (1992, Table 1), observations continued on the majority of the fields following publication of these preliminary results. Starting in late 1992, a camera employing a thinned, backside-illuminated Tektronix
$2048 \times 2048$ pixel CCD, with a flatness characterized by a raised hump of not more than $220 \mu \mathrm{m}$ peak to valley, was tested and commissioned. This so-called TEK2K camera provides a generous $11 \times 11$ arcmin FOV and, hence, a much richer selection of potential reference stars for the differential astrometric measures. Although the image sampling is somewhat degraded $\left(24.0 \mu \mathrm{m}\right.$ pixels giving $0.325^{\prime \prime}$ pixel $^{-1}$ ), good centroids were obtained. Consequently, the TEK2K camera rapidly became the workhorse for the NOFS parallax observations and the TI800 camera was formally decommissioned in 1995. The observational procedures employed with the TEK2K camera have been summarized in Dahn et al. (2002, Section 2.1).

After only a few years of experience using the TEK2K camera, it became apparent that relative parallaxes with uncertainties $\sim 0.5$ mas were routinely being achieved. This improvement can be attributed primarily to the much better reference star frames available using this larger FOV CCD. However, along with the improved relative parallaxes came the need for higher quality determinations for the correction from relative to absolute parallax - the quantity providing the actual distance determinations needed for astrophysical applications. (For additional information regarding this correction, see the footnote on page 1173 of Dahn et al. 2002). For the TI800 results presented in Monet et al. (1992), we - following YPC4 - employed their method of using the average magnitude of the reference stars employed in the field of each individual parallax target along with a galactic model to estimate the average reference star frame distance for each galactic direction. Although this approach was entirely adequate for the parallax determinations in YPC4, applying it for the higher precision TEK2K relative parallaxes would significantly inflate the uncertainties derived for the absolute parallaxes. Since we were already measuring $V, V-I$ photom- 
etry of each individual reference star employed in each parallax target field to calculate corrections for differential color refraction (DCR; see Monet et al. 1992, Section 3.2), we adopted a calibrated version of the $M_{V}$ versus $V-I$ colormagnitude diagram to determine photometric parallaxes for each star. (For further details on this calibration, see Harris et al. 2016.) Photometry from the 2MASS Point-Source Catalog (Skrutskie et al. 2006) is employed, when required, to resolve ambiguities in dwarf versus giant status of reference stars when the relative astrometry is not decisive and to clarify issues regarding interstellar extinction/reddening in fields at low galactic latitudes.

The higher precision TEK2K relative parallax determinations also necessitated an upgrade in the determination of parallax factors - the projected baseline along the Earth-Sun direction at each epoch of observation. These quantities enter the equations of conditions used in the least-squares solution for the parallax as multiplicative products with the parallax. Since the NOFS parallax determinations are mainly for stars at distances between 10 and 300 parsecs, parallax factors calculated to four significant figures are required. Hence, values calculated for the projected distance between the Earth and the solar system barycenter were adopted.

A third camera employing an EEV (English Electric Valve; now e2v) $2048 \times 4096$ pixel CCD42-80 thinned backside-illuminated CCD was commissioned in early 2008. This device has $13.5 \mu \mathrm{m}$ pixels, corresponding to $0.183^{\prime \prime}$ pixel $^{-1}$ on the sky. Hence, it provides better image sampling than TEK2K and gives an FOV of $6.2 \times 12.4$ arcmin on the sky. Furthermore, the pixels of this device have larger full-well charge capacity than the TEK2K chip, and this results in a slightly enhanced dynamic range before saturation effects become important. This detector had suffered a hygroscopic exposure to its anti-reflection (AR) coating while in use in tests supporting the ill-fated USNO Full-Sky Astrometric Mapping Explorer satellite engineering effort, resulting in discoloration of the backside surface when visibly inspected. The AR coating mostly returned to its manufactured state after six months of storage in a vacuum environment. The CCD's deviation from flatness is not more than $15 \mu \mathrm{m}$ peak to valley. The scheduled observing time on the Strand reflector around new moon then became shared between TEK2K and EEV24.

Another camera, employing an engineering grade Tektronix $2048 \times 2048$ CCD with a circular $(\sim 3 \mathrm{~mm}$ diameter, $\sim 40$ arcsec on the sky), highly uniform neutral density attenuation spot (Inconel on an optically flat quartz substrate), mounted $\sim 1 \mathrm{~mm}$ in front of the CCD, was commissioned in late 1995. The spot provides $\sim 9.0$ magnitudes of brightness reduction for the parallax target star, permitting targets as bright as fourth magnitude to be measured. The original motivation for this camera - designated ND9 - was concern about possible degradation in the astrometry from the ESA - it Hipparcos satellite due its failure of the craft to reach its intended geostationary orbit following launch in 1989 August. Happily, the final Hipparcos parallaxes obtained from the 4 year mission proved to be determined to the 1 mas level, as planned. Instead, a limited program was initiated with ND9 for the purpose of demonstrating that precision astrometry could be achieved from the ground using this form of magnitude compensation. 68 Hipparcos stars were observed over an epoch range of 5.2 years (on average) during the period from 1996 to 2002. A sample of preliminary results were presented in Harris et al. (1997). The derived ND9 parallaxes agreed well with the published values from Hipparcos, with parallax uncertainties generally better than their Hipparcos counterparts, except in a few cases. ND9 was decommissioned from routine parallax work in early 2002. 


\section{ASTROMETRIC RESULTS}

We continue to employ the image centroiding method developed by D. Monet (cf. Monet \& Dahn 1983, Section III.b) which employs an integral of the illumination under a circular symmetric Gaussian as the algorithm. Headto-head comparison with other techniques employed by DAOPHOT (Stetson 1987) and SExtractor (Bertin \& Arnouts 1996) show that the Monet method is at least as stable in uncrowded fields where uncontaminated images are encountered. In fields where the target star is blended with neighboring objects, DAOPHOT was used for deblending and centroiding. However, the Monet method is not appropriate for photometric reductions and is not employed for those observations (see Section 4 below).

Our solutions for parallax are carried out independently in both RA and DEC. These values are then combined as a weighted average to obtain the final value presented below. The validity of this approach was discussed by Lutz \& Upgren (1980), and offers the advantage of identifying bad data (e.g., a cosmic-ray strike in the wings of an image) and providing an alert to possible astrometric perturbations.

The astrometric results are presented in Table 1 . These determinations include all acceptable observations obtained through 2016 June and employ all of the reduction improvements outlined in the previous section. Results for a total of 309 individual stars are tabulated with two independent determinations included for four stars (LP 540-16, LHS 2397a, LHS 3406, and LHS 474). Column (1) in Table 1 gives the identifying number in the 2MASS Point Source Catalog (Skrutskie et al. 2006). The 2MASS J number provides an unambiguous link to SIMBAD where many alternate names and much additional information can be found. A few stars are not individually found in 2MASS, usually due to their proximity to another star at the epochs of the 2MASS observations. For these stars, we adopt their LHS designation in column (2), and their coordinates from Gaia DR1 (Lindegren et al. 2016).

Since the majority of the stars in Table 1 were originally identified due to their large proper motions found in the surveys carried out at the University of Minnesota (Luyten 1964) and Lowell Observatory (Giclas et al. 1971, 1978), researchers in fields studying nearby stars are often familiar with them by their survey designations. Column (2) of Table 1 gives a common name for the parallax star, and since 197 of them are included in Luyten's LHS Catalog (Luyten 1979), that designation is given preference. For 16 proper motion survey stars with annual motions less than $0.4^{\prime \prime}$ Luyten numbers (LP or NLTT) or Lowell numbers $(\mathrm{G})$ are used. The names for the remaining stars are explained in the Notes to Table 1. For stars for which an earlier (preliminary) NOFS parallax was presented in either Monet et al. (1992) or Dahn et al. (2002), a table note with reference can be found in Table 1. We emphasize that the determinations presented here are not independent of the previous NOFS results but rather supersede them.

Column (5) indicates the camera employed for each parallax determination. Values of 1,2 , and 3 correspond to the TI800, TEK2K and EEV24 cameras, respectively. Column (6) indicates the filter used. ST-R is the STWIDER filter whose properties were described in Monet et al. (1992, Section 2.2). A2-1 is an optically flat interference filter that defines a bandpass centered near $698 \mathrm{~nm}$ and with an FWHM of approximately $172 \mathrm{~nm}$. I-2 is another optically flat interference filter that defines a bandpass centered near $810 \mathrm{~nm}$ and with an FWHM of approximately $191 \mathrm{~nm}$. Z-2 is an optically flat $3 \mathrm{~mm}$ thick piece of Schott RG830 glass producing a relatively sharp blue-edge cutoff near $830 \mathrm{~nm}$. The actual bandpass depends heavily on the sensitivity of the CCD. The bandpass closely ap- 
Table 1. Astrometric Results

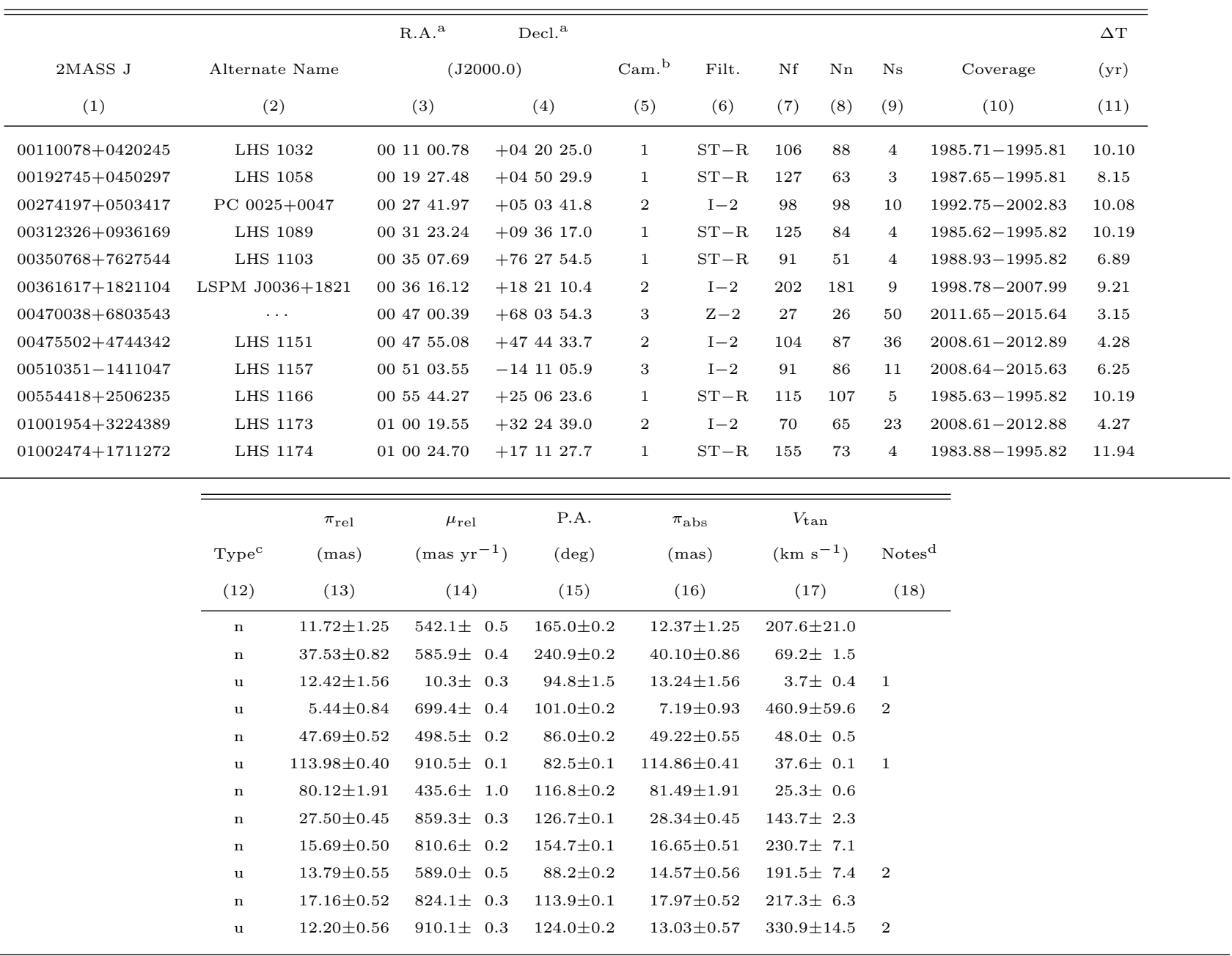

NoтE-Table 1 is published in its entirety in machine-readable format. A portion is shown here for guidance regarding its form and content.

${ }^{a}$ Coordinates are from the 2MASS catalog when available, else from Gaia Data Release 1, propagated to epoch J2000 using proper motions presented here.

${ }^{b}$ Camera: (1) TI800, (2) TEK2K, (3) EEV24

$c$ Type of astrometry: (n) New results (previously unpublished). (u) Update of previously published results, using additional observations. (r) Revision of previously published results using parallax factors with respect to the solar system barycenter and revised corrections from relative to absolute parallax, as described in Section 2.

$d$ Notes on individual objects: (1) Previous astrometric results were published in Dahn et al. (2002). Results presented here arise from updated reduction methods and may include additional data, thus, they supercede the results of Dahn et al. (2002). (2) Previous astrometric results were published in Monet et al. (1992). Results presented here arise from updated reduction methods and may include additional data, thus, they supercede the results of Monet et al. (1992). (3) Object is a field reference star for LHS 3259 for which there were measurable astrometric results. It was named LHS 3259.1 in Monet et al. (1992). (4) Object is a field reference star for LHS 3548 for which there were measurable astrometric results. It was named LHS 3548.1 in Monet et al. (1992).

proximates the SDSS $z^{\prime}$ bandpass (cf. Fukugita et al. 1996; Smith et al. 2002). Figure 1 shows the filter transmissivities measured by the manufacturer along with the quantum efficiencies of the TEK2K and EEV24 CCDs supplied by the manufacturers. The difference between the TEK2K and EEV24 responses is noticeable and, hence, we did not mix observations taken with different cameras nor did we mix frames taken with different filters when performing the astrometric reductions. These filters are mounted in a tray that is part of the autoguider assembly, placing them approximately $7 \mathrm{~cm}$ in front of the CCDs and assuring that the out-of-focus image of each star will be insensitive to any slight filter irregularities.

Columns (7), (8), and (9) in Table 1 give the number of acceptable CCD frames (observa- 


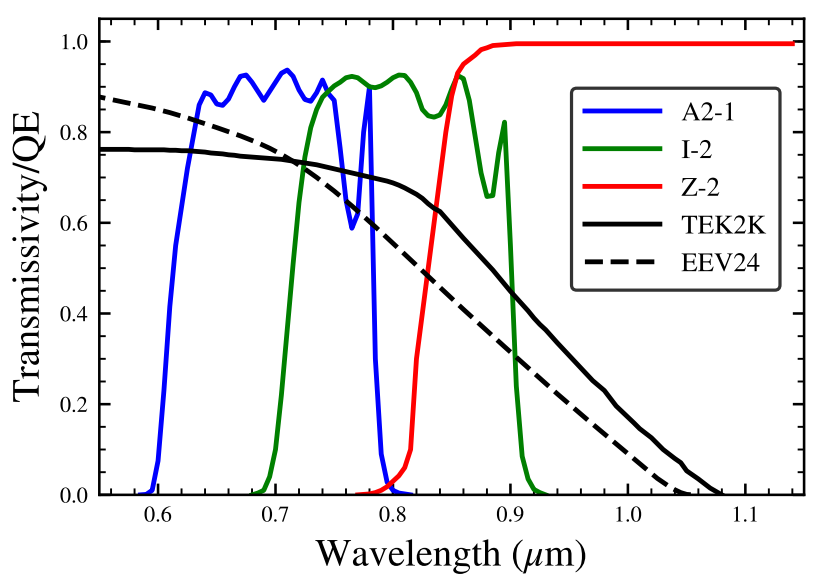

Figure 1. Transmissivity of the filters, and quantum efficiency of the CCDs, used in the NOFS parallax program.

tions), the number of separate nights on which those observations were obtained, and the number of reference stars employed in astrometric solutions, respectively. Real-time reduction to centroids is performed while the observing is in progress, immediately after the camera shutter closes. Processing is done for a large selection of potential reference stars which were designated when the field was originally added to the program. Once photometry has been obtained for the field (parallax target star and all reference stars), then we carefully decide which of the potential reference stars will actually be employed. Consideration of the frame configuration and reliability of determining the correction from relative to absolute parallax (see below) are paramount. Columns (10) and (11) give the years observed and total epoch range, respectively. Column (12) specifies whether this is a new result (previously unpublished), an update of a previously published result using additional data, or a revision of a previously published result using parallax factors relative to the solar system barycenter and a revised correction from relative to absolute parallax, as described in Section 2.

The derived relative parallax and its mean (standard) uncertainty are given in Column (13) (throughout this work we refer to the trigonometric parallax angle as $\pi$ ). The relative total proper motion and its uncertainty follow in Column (14), and the position angle of the proper motion is given in Column (15). Our formal solution for the position angle is often precise to $0.02-0.04$ degrees. However, the reduction of each field's ensemble of CCD frames to a standard frame does not include the uncertainty in the orientation of each CCD in its dewar plus an additional small uncertainty introduced when the dewar is taken on/off the telescope during monthly instrument changes. The orientation of the chip columns (or in the case of the TI800, rows) to rigorously align with eastwest on the sky was carried out by taking star trails with the telescope pointed at the celestial equator while stationary on the meridian. This time-consuming, iterative procedure was carried out when the cameras were first commissioned. From then on, scribe marks on the dewar-tomounting plate interface were employed for reorientation following instrument changes. Variations in solution plate (frame) constants indicate that quoting the uncertainty in position angle (Column (15) of Table 1) to only \pm 0.1 degree safely accounts for any systematic chip orientation issues.

Figure 2 shows the frequency distribution for the uncertainties of the relative parallax determinations, including a breakdown for the three separate cameras. All three cameras produced relative parallaxes with formal precisions in the $\pm 0.3-0.7$ mas range when $\gtrsim 30$ observations spanning an epoch range of $\gtrsim 4$ years were employed. The extended tails toward larger uncertainty in the Figure 2 distributions for the TEK2K and EEV24 cameras primarily result from very faint targets or very bright targets where the exposure times are too short to properly average out atmospheric effects. The more pronounced excess of larger uncertainties for the TI800 fields is primarily due to very poor refer- 


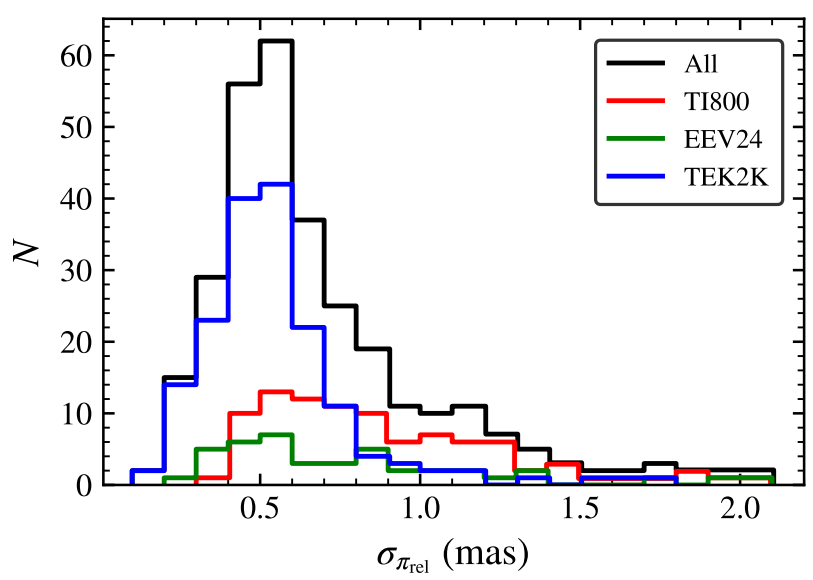

Figure 2. Distribution of the relative parallax uncertainties, for all stars in this paper (black histogram), and for stars observed with the individual cameras.

ence star frame configurations available in that camera's limited FOV.

The derived absolute parallax and its uncertainty are presented in Column (16). The correction from relative to absolute parallax was carried out using the procedures described in detail by Harris et al. (2016). In summary, a photometric parallax is derived for every reference star employed using a calibrated $M_{V}$ vs $V-I$ diagram constructed from large, accurate Hipparcos parallaxes for stars with $M_{V}<$ 13, plus stars with $M_{V}>9$ based on accurate USNO photographically measured parallaxes. Photometry measured at NOFS on the Johnson-Kron-Cousins system is employed (see Section 4). For fields located at lower galactic latitudes, other reference star photometry (2MASS $J H K_{s} ; B V I$ from various sources) is employed to help resolve dwarf/giant ambiguities and improve estimates for interstellar reddening/extinction corrections.

The calculated tangential velocities and their uncertainties are given in Column (17) of Table 1 . We have not attempted to convert from relative to absolute proper motions. Such corrections are very uncertain, due to necessary assumptions regarding galactic rotation (see Har-

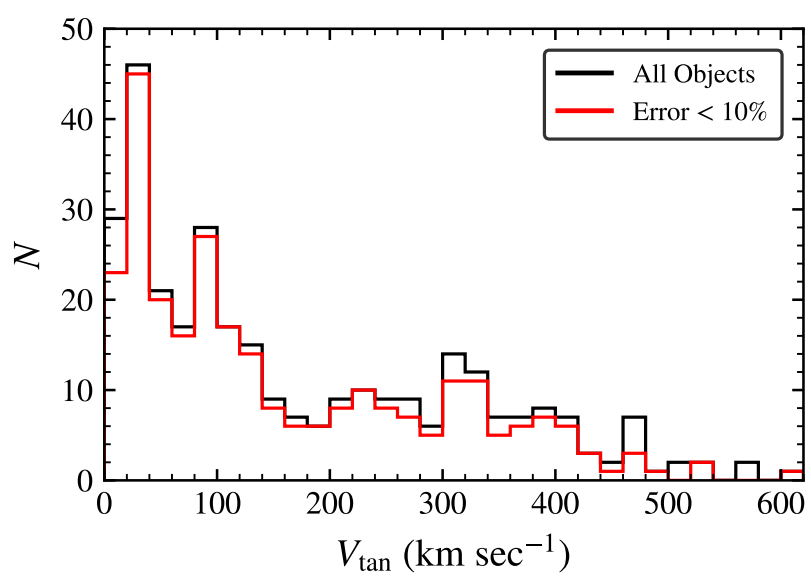

Figure 3. Distribution of tangential velocities.

ris et al. 2016, Section 3.3). However, for the faint reference stars employed for the parallax determinations presented here, these corrections amount only to $1-3$ mas $\mathrm{yr}^{-1}$. Since all but 19 of the stars in the present sample have relative proper motions greater than 100 mas $\mathrm{yr}^{-1}$, the use of $\mu_{\text {rel }}$ in calculating $V_{\text {tan }}$ has little effect. Figure 3 shows the frequency distribution of $V_{\tan }$ for the Table 1 sample. Note that fully one-third of the stars in the Table 1 sample have $V_{\tan }>200 \mathrm{~km} \mathrm{~s}^{-1}$. This is, of course, not surprising since our sample is highly biased toward higher tangential velocities since targets were given priority to being added to our parallax program if we knew or suspected that they might be subdwarfs.

\subsection{Individual Stars}

Many of the stars in Table 1 are among the most intensely studied objects in the nearby solar neighborhood. It is beyond the scope of the present paper to review the extensive literature for them. The interested reader is referred to relevant references in SIMBAD. However, some brief notes concerning our Table 1 astrometry for a few stars follows.

$\mathrm{PC} 0025+0447$ and 2MASS J16241436+0029158 were both observed with the I-2 filter before the Z-2 filter became available. The large uncertainties of the 
relative parallaxes for these two stars reflects the marginal exposure level of these very red and faint targets.

2MASS J00470038+6803543 has a large relative parallax uncertainty, the direct result of the faintness of this object even at $Z$-band. Observations could only be made in subarcsec seeing and on nights with clear, dark sky.

LHS 1252 has a very poor available reference star frame, necessitating the use of one very faint star and a faint galaxy.

GAT $\mathbf{1 3 7 0}$ has a preliminary distance estimate $-d=2.4(+0.7,-0.40)$ pc - derived by Teegarden et al. (2003) and the star is often referred to as "Teegarden's star." The weighted mean of five independent parallax determinations (see Table 2 below) places this star at a distance of $3.842 \pm 0.004 \mathrm{pc}$, making it the $23 \mathrm{rd}$ nearest non-BD star (system) from the sun.

\section{MASS J04193697+1433329,}

2MASS J04223075+1526310, and 2MASS J04325119+1730092 were suggested as possible brown dwarf members of the Hyades cluster (Leggett \& Hawkins 1989) but were shown by NOFS astrometry (Harris et al. 1999) and spectroscopy (Reid \& Hawley 1999) to be pre-main-sequence stars in the background of the cluster.

2MASS J04433761+0002051 was suggested as an ultracool late M-dwarf member of the AB Doradus moving group by Schlieder et al. (2012). Earlier, Faherty et al. (2009) had proposed it as a member of the $\beta$ Pictoris group and Gagné et al. (2014) concurred. The parallax determination by Liu et al. (2016) supports the $\beta$ Pictoris membership. Our Table 1 results are in good agreement (see Table 2 below) and adds further strength to that interpretation.

2MASS J04435686+3723033, the BY Draconis variable V961 Per, was identified as a likely member of the $\beta$ Pictoris moving group by Schlieder et al. (2010) who gave an estimated kinematic distance of 76.9 pc.
Our Table 1 parallax translates to a distance of $73.7(+2.6,-2.3)$ pc, in satisfactory agreement with their prediction. Schlieder et al. (2010) also report a faint common proper motion companion located $\sim 9^{\prime \prime}$ to the east of $2 \mathrm{M} 0443+3723$. We performed a solution using 23 uncontaminated exposures of the companion, obtaining $\pi_{\mathrm{abs}}=14.41 \pm 1.31$ mas and $\mu_{\text {rel }}=64.5$ mas $\mathrm{yr}^{-1}$ at P. A. $=158.0 \pm 0.5^{\circ}$. Both strongly support the physical nature of the pair. At epoch 2015.053 we measure $\rho=7.68^{\prime \prime}$ at P. A. $=91.65^{\circ}$.

LSPM J0602+3910, an L1V star, was maintained on the observing program for over 12 years to monitor the stability of our astrometric results and to look for any detectable astrometric perturbation. The relative parallax uncertainty of 0.19 mas is the third best achieved by the CCD program. The formal residuals from the solution in each coordinate (Figure 4, Panel a) show no convincing evidence of an astrometric perturbation and demonstrate the stability of residuals derived from observations spanning epoch ranges as long as a decade.

LHS 1839 has a proper motion which has carried it toward a brighter field star. During the epochs of our astrometric measures (1986.23 to 1995.16) they were still well enough separated to yield uncontaminated measures when the seeing was $\sim 1.5^{\prime \prime}$ FWHM or better.

LP540-16 has a proper motion $>1.0^{\prime \prime} \mathrm{yr}^{-1}$, first announced by Luyten \& Hughes (1983), and was added to the NOFS TEK2K program in 2003 March. It was removed as completed in 2008 March. Lépine et al. (2002) announced (what turned out to be) a "rediscovery" of the same star, and it was inadvertently added to our EEV24 program in 2012 December. When the repeat was discovered in 2014 March, it was again deactivated. The excellent agreement between the two totally independent determinations is reassuring. Thus, a weighted mean of $14.55 \pm 0.23$ mas is appropriate. 


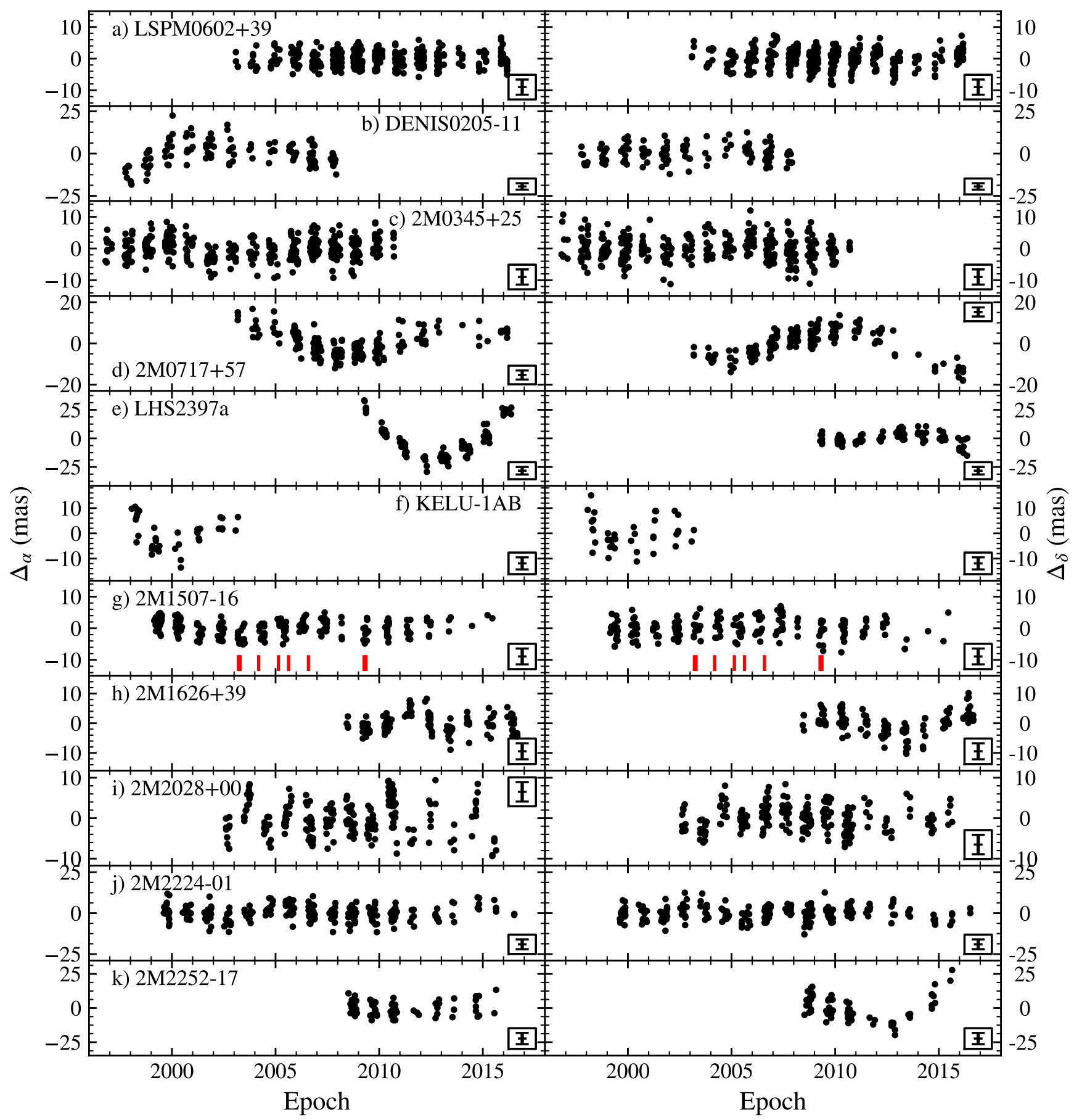

Figure 4. Astrometric residuals for individual stars discussed in the text. The left panels show the residuals in right ascension, while the right panels show the residuals in declination. Typical error bars are indicated on the right-hand side of each panel. For 2MASS J15074769-1627386 (Panel g), the red lines indicate the epochs of the radial velocity measures of Blake et al. (2010). 
LHS 2471 has a very large uncertainty in the parallax determination, primarily due to the extremely poor reference frame available.

LHS 2557 was observed over two intervals from 1993.07 to 1997.29 and then again from 2001.28 to 2008.17 - due to the star's proper motion carrying it across a faint field star.

LHS 453 has a revised solution in Table 1 employing a tighter 4 star reference frame and over twice the number of observations as were available for the preliminary parallax determination presented in Monet et al. (1992).

LSPM J1826+3014 has a proper motion that carried this star across a field galaxy making the target star unobservable between 2005.37 and 2008.31.

LHS 3406, observed with TI800 where a rich but large reference star frame was available, was also observed using TEK2K as a consistency check. As Table 1 shows, the two independent absolute parallax determinations agree very well with each other to within the combined uncertainties. Thus, a weighted mean of $69.09 \pm 0.38$ mas is appropriate.

LHS 474 is vB10 and our preliminary parallax determination was presented in Monet et al. (1992). Continued observations were complicated by vB10's proper motion carrying it across a moderately bright field star, and it was removed from the program on $2009 \mathrm{Au}-$ gust 21. The residuals from that parallax solution showed no sign of a perturbation in either RA or DEC. When the astrometric discovery of a purported candidate planet was announced (Pravdo \& Shaklan 2009), vB10 was added to the EEV24 program. Subsequently, Bean et al. (2010) presented precision $( \pm 10$ $\mathrm{m} \mathrm{s}^{-1}$ ) radial velocity measures spanning $\sim 225$ days in 2009 and found no variation, concluding that the proposed 6.4 Jupiter-mass planet in a $P=0.744 \mathrm{yr}$ orbit is "spurious." Our EEV24 observations now span the 7.06 yr interval from 2009 June 15 to 2016 July 5. How- ever, vB10 passed very close to another field star in the 2010 - 2011 interval. Nonetheless, these observations show no indication of the Pravdo \& Shaklan perturbation. However, as Pravdo \& Shaklan themselves noted, this field is "an astrometrist's dream in a nightmarish setting." On the positive side, it's location at $b=-3.3^{\circ}$ provides a plethora of potential reference frame stars. On the negative side, at least for the parallax determination, is that its location near the galactic equator presents problems with interstellar reddening/extinction in determining the correction from relative to absolute parallax. Furthermore, vB10's large proper motion through such a crowded field presents potential for contamination at repeated epochs. At this time, we cannot reject such possibilities and are continuing our observations.

\section{LHS 3548 and}

2MASS J20253876-0612094 have updated TI800 astrometry employing a very tight 5 star reference frame. Note that the latter was referred to as LHS 3548.1 in Monet et al. (1992) because it was found to have a measurable parallax serendipitously while serving as a reference star.

LSPM J2036+5100 has an incorrectly reported position angle for the proper motion of $100.8^{\circ}$ in Lépine et al. (2003).

LHS 3684 uses only the RA parallax solution in Table 1, due to some contamination from a faint star $\sim 2^{\prime \prime}$ due south of the target. Additionally, we suspect the quoted proper motion might also be affected.

2MASS J21374019+0137137 was suggested as a likely member of the $\beta$ Pictoris moving group by Schlieder et al. (2012). Treating apparent radial velocity variations as due to an SB2 led to an unphysical interpretation. In our good seeing observations $\left(\leq 0.8^{\prime \prime}\right.$ FWHM) the images of $2 \mathrm{M} 2137+0137$ seem to be slightly distorted when compared to nearby field stars. This suggests that it might be a close binary 
where the faint companion has a separation of $\sim 0.5^{\prime \prime}$.

2MASS J22004158+2715135 was observed at the request of J. Schlieder for his studies of moving group membership candidates. Because of the brightness of the target $(I=9.59)$, exposure times were restricted to $<20 \mathrm{~s}$ in good seeing. Consequently, the averaging over atmospheric effects was inadequate, and the resulting uncertainties in the solution reported in Table 1 are large. In seeing $\leq 0.8^{\prime \prime}$ FWHM a faint star is detected roughly due north of $2 \mathrm{M} 2200+2715$. At epoch 2012.712 we measure it at $\rho=5.34^{\prime \prime}$ and P. A. $=357.2^{\circ}$. Common proper motion has not yet been established for the two stars.

LHS 541 is vB12, a companion to LHS 540 $=\mathrm{BD}-14: 6437 \mathrm{AB}$ at $\rho \sim 15^{\prime \prime}$ and P. A. $\sim 47^{\circ}$. The Table 1 solution is restricted to observations taken in $\leq 1.6^{\prime \prime}$ FWHM to preclude contamination from LHS 540.

LHS 3937 was found by Luyten (1980) to

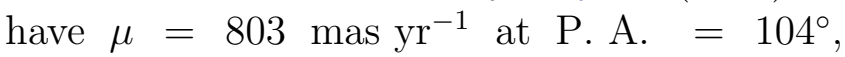
whereas Salim \& Gould (2003) reported $\mu=$

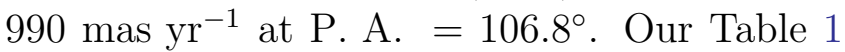

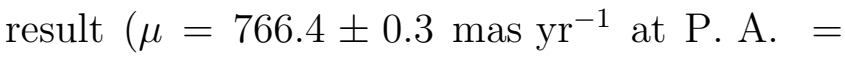
$\left.104.9 \pm 0.1^{\circ}\right)$ is in closer agreement with that of Luyten.

\subsection{Resolved Binary Systems - Physical and Optical}

Several of the stars in Table 1 are members of binary systems whose components are separated enough to be resolved in our observations. Comments on them follow.

LHS 1203 and LHS 1205 form a common proper motion pair with separation $\rho \sim 7.9^{\prime \prime}$ at P. A. $\sim 133.6^{\circ}$. Bakos et al. (2002) reported significantly different proper motions for the two. Our astrometry confirms the physical nature of the pair.

PG 0235+064B was discovered as a common proper motion companion to the DA3.5 white dwarf PG0235+064 = GJ3173 = 2MASS
J02383078+0638071 in the course of our astrometric observations of the WD. For the WD we measure $\pi_{\text {rel }}=16.03 \pm 0.33$ mas, $\mu_{\text {rel }}=$

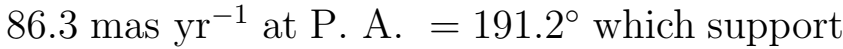
the physical nature of the pair.

LHS 189 and LHS 190 form a close physical binary $\left(\rho \sim 1.2^{\prime \prime}\right.$, P. A. $\left.\sim 146.2^{\circ}\right)$ which required DAOPHOT centroiding of the targeted stars to avoid contamination between the two. The fainter of the two (LHS 190) could not be measured on 34 of the frames suitable for the brighter component (LHS 189).

LHS 2099 and LHS 2100 form a common proper motion pair with $\rho=6.66^{\prime \prime}$ and P. A. $=$ $100.8^{\circ}$. LHS 2100 is approximately $2.8 \mathrm{mag}$ fainter than LHS 2099 in the STWIDER-band used in our astrometry, resulting in consistently weakly exposed images. This accounts for the unusually large uncertainty in our parallax determination. Nevertheless, our astrometry is good enough to confirm the physical status of the system.

LHS 2140 is the bright $(V \sim 15.04)$ primary component of a physical binary system with LHS 2139 ( $V \sim 19.6$; spectral type DC? from Gizis \& Reid 1997b). From observations obtained in good seeing we measure $\rho=6.7^{\prime \prime}$, P. A. $=28.9^{\circ}$. From 100 frames suitable for measurement of the fainter component we find $\pi_{\text {rel }}=13.73 \pm 1.40$ mas and $\mu_{\text {rel }}=580.0 \pm$ $0.7 \mathrm{mas} \mathrm{yr}^{-1}$ at P. A. $=186.9 \pm 0.7^{\circ}$. The system is clearly physical.

LHS 6176 is the bright component in a common proper motion binary system containing a T8p secondary star $\sim 52^{\prime \prime}$ away (Luhman et al. 2012; Burningham et al. 2013). The secondary is much too faint in the optical for us to observe.

LHS 2444 and LHS 2445 form a common proper motion pair with $\rho=10.74^{\prime \prime}$ and P. A. $=137.6^{\circ}$. Our astrometry confirms the physical status of the system.

G166-37 (= Ross $50=$ LP381-87) and LP 381-86 form a widely separated common 
proper motion pair. We measure $\rho=200.8^{\prime \prime}$ at $\mathrm{P} . \mathrm{A} .=205.8^{\circ}$ which, along with our parallaxes, confirms the physical status of the system at the 1.5-sigma level.

LHS 3001 and LHS 3002 are clearly a physical system. Due to the faintness of the secondary (LHS 3002) astrometry could not be obtained from 11 of the frames employed for LHS 3001.

\section{LSPM J1457+2341S}

(=2MASS J14572616+2341227), located 2.3" east and $\sim 3.2^{\prime \prime}$ south of the dwarf carbon star candidate SDSS 1457+2341 (=2MASS $\mathrm{J} 14572597+2341257$ ) (Green 2013) is a possible - but far from certain - physical companion. Being 2.3 mag fainter than the $\mathrm{dC}$ star at $I$-band and only $\sim 4^{\prime \prime}$ distant, it is difficult to measure. For the brighter component we measure $\pi_{\text {rel }}=7.42 \pm 0.55$ mas and $\mu_{\text {rel }}=$

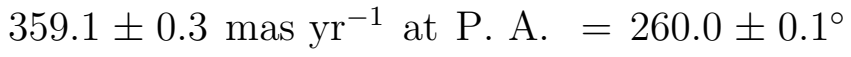
from 35 observations. Although not definitive, the close similarity of our values is strongly suggestive. Recently, Kirkpatrick et al. (2016) announced this as a candidate physical triple system based on data from the ALLWISE motion survey. Our observations are continuing.

LHS 3181 appeared to be a binary on several exposures taken in very good seeing. The potential companion is faint $\left(\Delta_{r}=3.73 \pm 0.03 \mathrm{mag}\right.$ in the ST-R bandpass) and located at $\rho \sim 3.56^{\prime \prime}$ and P. A. $\sim 155.4^{\circ}$ (epoch 1991.44). Observations continuing until 1995.57 confirmed that this faint star is a background field star.

2MASS J17054834-0516462 appeared to have a binary companion with $\rho \sim 1.36^{\prime \prime}$ and P. A. $\sim 5^{\circ}$ in NICMOS exposures on HST (Reid et al. 2006a). However, Andrei et al. (2011) concluded from photometry that the faint star was most probably a more distant ( 200 pc) late M dwarf. Our results in Table 1 are from DAOPHOT centroids of the 2MASS target. Attempts to measure the fainter star were only marginally successful. Employing 84 acceptable observations made in better seeing, we find a proper motion that is immeasurably small and an absolute parallax of $6.3 \pm 4.9$ mas, a value that confirms it is not a physical companion.

2M19302746-1943493 has a faint star nearby for which we estimate $\rho \sim 1.5^{\prime \prime}$, P. A. $\sim$ $\mathrm{SSW}$, and $\Delta_{I}=0.8 \mathrm{mag}$. With the field located at a large zenith distance, the pair is often significantly blended on our frames. DAOPHOT centroiding was employed for both stars to determine whether they form a physical pair. For the fainter star we find $\pi_{\text {rel }}=39.34 \pm 1.67$ mas

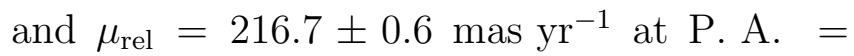
$104.5 \pm 0.2^{\circ}$. Allowing for the problem with image blending, it appears that they do form a physical pair. Based on our estimated value of $\Delta_{I}=0.8$, we infer that the $\mathrm{B}$ component is most likely an $\sim \mathrm{M} 8 \mathrm{~V}$ companion to the M6.5Ve primary component.

LHS 5359 and LHS 5360 are clearly a physical system. The fainter component (LHS 5360) is located $\sim 2.5^{\prime \prime}$ away from the considerably brighter component and is measurable on only $\sim 43 \%$ of the frames acceptable for LHS 5359 .

\subsection{Unresolved Binary Systems - Old and New}

Table 1 includes 12 binaries for which we can not resolve the individual components from our ground-based observations. Four are previously unrecognized systems.

DENIS J0205.4-1159AB was discovered to be a close physical binary pair by Koerner et al. (1999). Using a collection of data from various sources, Bouy et al. (2003) estimated the period to be $\sim 75 \mathrm{yr}$. The residuals from our astrometry (Figure 4, Panel b) cover 10.17 years over the epoch range from 1997.8 to 2007.9 and show a possible inflection (nonlinearity) in RA starting around 2001.

2MASS J03454316+2540233 was observed to be a double-lined spectroscopic binary by Reid et al. (1999). The residuals from 
our 13.91 yr of observational coverage (Figure 4, Panel c) show some evidence for a lowamplitude perturbation in the residuals with an estimated period of $\sim 7.7 \mathrm{yr}$. However, the evidence is admittedly weak and observations are not being continued on this field.

2MASS J07171626+5705430 exhibits residuals from our solution for parallax and proper motion clearly indicating a perturbation in both RA and DEC (Figure 4, Panel d). Since we apparently have not observed a full period yet, all we can say for sure is that $P>10 \mathrm{yr}$.

2MASS J08503593+1057156 is the star selected by Kirkpatrick et al. (1999) to define the L6V spectral class. Notable was the presence in the spectrum of strong $(\mathrm{EW} \sim 15 \AA$ ) Li $\lambda 6708 \AA$ absorption. HST images of the star by Reid et al. (2001) revealed that it is a tight binary with $\rho \sim 0.16^{\prime \prime}$, P. A. $\sim 250^{\circ}$, and $\Delta_{I} \sim 1.3 \mathrm{mag}$. They also noted the presence of a slightly brighter field background $\mathrm{M}$ dwarf located approximately due east of $2 \mathrm{M} 0850+1057$ with $\rho<2^{\prime \prime}$ on 2000 February 1 Feb. The preliminary NOFS CCD parallax $\pi_{\text {abs }}=39.1 \pm$ 3.5 mas presented in Dahn et al. (2002) was based on 30 observations taken between 1997 December 4 and 2001 March 29. A preliminary NOFS near-infrared parallax based on 13 observations with a mean epoch of 2001.79 found $\pi_{\text {abs }}=26.22 \pm 4.21$ mas (Vrba et al. 2004) and the discrepancy between the two determinations was noted. Faherty et al. (2011) have argued quite convincingly that this poor agreement is most likely due to contamination in the earlier CCD observations from the field $\mathrm{M}$ dwarf which we measure to have small relative proper motion such that $2 \mathrm{M} 0850+1057$ is moving away from

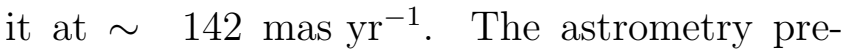
sented here in Table 1 was obtained by rejecting all observations taken prior to 2001 February 17 and all observations where the seeing was greater than 1.4" FWHM. Unfortunately, this left only 25 acceptable frames and the large uncertainty in the derived relative parallax reflects the paucity of observations for this very faint target. However, our CCD determination is now in satisfactory agreement with both the improved NOFS near-infrared parallax and with the near-infrared parallax reported by Dupuy \& Liu (2012). See Table 2 below.

$2 \mathrm{M} 09153413+0422045$ is a pair of L7V stars with $\rho=0.73^{\prime \prime}$ (Reid et al. 2006a). Attempts to measure both stars from frames obtained in very good seeing were not successful. Using only frames obtained in moderate seeing (generally, $1.2-1.8^{\prime \prime}$ FWHM), where the pair is well blended, yielded the results reported in Table 1.

LHS 2397a was announced to have an unresolved brown dwarf companion by Freed et al. (2003). Our TI800 parallax for this star was presented as a completed determination well before this (Monet et al. 1992). The large parallax uncertainty in that result is mostly a consequence of the very poor reference star frame available, necessitating the use of a very faint galaxy. The field was added to our EEV24 program (where it benefits from a much more favorable reference star frame) in 2009 April. With $P \sim 14.2$ years (Dupuy et al. 2009; Konopacky et al. 2010), we have observed it to date for less than half of a full orbital period. However, the residuals from our parallax solution shows clear evidence for an astrometric perturbation, especially the RA coordinate (Figure 4, Panel e). The large (and growing!) uncertainty for the relative parallax presented in Table 1 reflects our current inability to remove the perturbation from our EEV24 distance determination. Dupuy \& Liu (2017) recently analyzed their measures of the photocentric motion for this system and derived a mass ratio of $0.71 \pm 0.03$ for the components.

Kelu-1AB was identified as a physical pair of L dwarfs by Liu \& Leggett (2005) using laserguided adaptive optics on the Keck II Tele- 
scope. In the near-infrared they measured $\rho=$ $0.291 \pm 0.002^{\prime \prime}$ and P. A. $=221.2 \pm 0.6^{\circ}$. A roughly estimated period of 35 years was obtained, although a much larger range of possible periods $(\sim 15-105$ years $)$ was acknowledged. Our observations span only 5.12 years and the residuals from the parallax/proper motion solution are shown in Figure 4, Panel $\mathrm{f}$. There is a suggestion of an astrometric perturbation in our data. However, the meridional crossing zenith distance of the field is over $60^{\circ}$, the total number of acceptable observations is small, and the TEK2K camera was employed at $I$-band (resulting in many weak exposures), so the possibility of a false perturbation can not be ruled out. The rather poor agreement between the three currently available parallax determinations for this system can be seen in the Table 2 compilation below.

2MASS J15074769-1627386 was added to our TEK2K program on 1999 February 23 and the residuals from our parallax solution in RA show the suggestion of a low-amplitude perturbation (Figure 4, Panel g). Inflections (nonlinearities) in the years 2003, 2008, and 2012 suggest an orbital period of $\sim 9-10$ years. Seven high-precision (rms error $\pm 0.27 \mathrm{~km} \mathrm{~s}^{-1}$ ) infrared radial velocity measures by Blake et al. (2010) showed a statistically significant linear variation of $\sim 0.5 \mathrm{~km} \mathrm{~s}^{-1}$ over the time integral 2003.20 to 2006.58. Then, three measures between 2009.26 and 2009.36 indicated either a flattening of this variation or possibly a reversal. These authors suggest that such behavior - if real - would indicate the presence of a low-mass companion in an orbit with $P>13$ years. The time coincidence of their event with ours motivates us to continue monitoring this target. Our observations now cover an epoch range of 16.3 years and predict that another inflection should occur in the 2016 - 2017 time frame. The large parallax which we measure for this star should not be significantly affected by a potential perturbation this small.

2MASS J16262034+3925190 is the only sdL observed on the NOFS optical parallax program. Residuals from the parallax solution show evidence for a low-amplitude perturbation in both RA and DEC (Figure 4, Panel h). The period appears to be in the $8 \pm 1$ year range but since our observations only cover an epoch range of 8.07 years, that is very uncertain. Observations are continuing on this field.

2MASS J20282035+0052265 displays evidence for a low-amplitude, short-period $(\lesssim 2 \mathrm{yr})$ perturbation in both the RA and DEC residuals to the parallax solution (Figure 4, Panel i). Observations are continuing. Gaia will easily confirm or refute the reality of the perturbation.

2MASS J22244381-0158521 was added to our TEK2K program on 1999 February 23 and observations have continued for $16+$ years now. The residuals from the parallax solution presented in Table 1 (Figure 4, Panel j) show evidence for a low-amplitude perturbation in both RA and DEC with an estimated period of $10-$ 12 years. Our observations are continuing.

2MASS J22521073-1730134 was resolved in HST NICMOS images by Reid et al. (2006b) as an L+T-type binary system with $\rho=0.130 \pm$ $0.002^{\prime \prime}$ at P. A. $=-9.6 \pm 1.2^{\circ}$. They estimate that the period would lie somewhere in the range $3-38$ years. Our observations cover an epoch range of $7.1 \mathrm{yr}$ and show definite evidence for a perturbation in DEC (Figure 4, Panel k). Observations are continuing.

\subsection{Comparison with Other Parallax Determinations}

Trigonometric parallax determinations have already appeared in the literature for 124 of the stars in Table 1. Table 2 presents a compilation of these results for comparison. The largest overlap with our Table 1 determinations is with MEarth (Dittmann et al. 2014) where we have 33 stars in common. Since the published proper 
motion determinations for MEarth did not include estimated errors for each star individually,

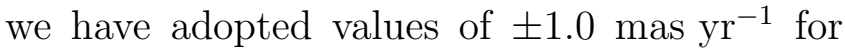
each coordinate based on advice from Dittmann (2016, private communication). The second largest overlap is with USNO photographically determined parallaxes, numbering 25 stars in common. (A compilation of all USNO photographic values can be found in Vol.II of YPC4.) Figure 5, Panel a shows a comparison of the values for these two subsets with our CCD values. The stars with USNO photographic determinations are some of the faintest targets attempted at NOFS. The parallax uncertainties are large, with a median value of \pm 4.2 mas. Examination of Figure 5 shows that most of the two independent USNO determinations agree to within $1.5 \sigma$ of the combined uncertainty in the difference, and hence, support the reliability of USNO photographic parallaxes at the precision level published.

The agreement between USNO CCD determinations and the MEarth results for the 33 stars in common is much less satisfactory. Figure 5, Panel a shows evidence for systematic differences in the sense that the MEarth parallaxes for this subsample are larger than our CCD determinations. Dittmann et al. (2014) estimate that for their full 1057 star sample they are realizing an average precision per star of 5 mas. The cataloged results for this 33 star subset give an average uncertainty for their absolute parallaxes of 4.3 mas. However, there are 15 stars in Figure 5 where the MEarth parallax is larger than the USNO value by over 8 mas, and as large as 26 mas.

Figure 5, Panel b shows our Table 1 absolute parallax determinations compared with the published results from the RECONS/CTIOPI Programs (Costa et al. 2005, 2006; Jao et al. 2005, 2011; Henry et al. 2006; Riedel et al. 2010; Dieterich et al. 2014) and the Torino Observatory Parallax Program (TOPP; Smart et al.

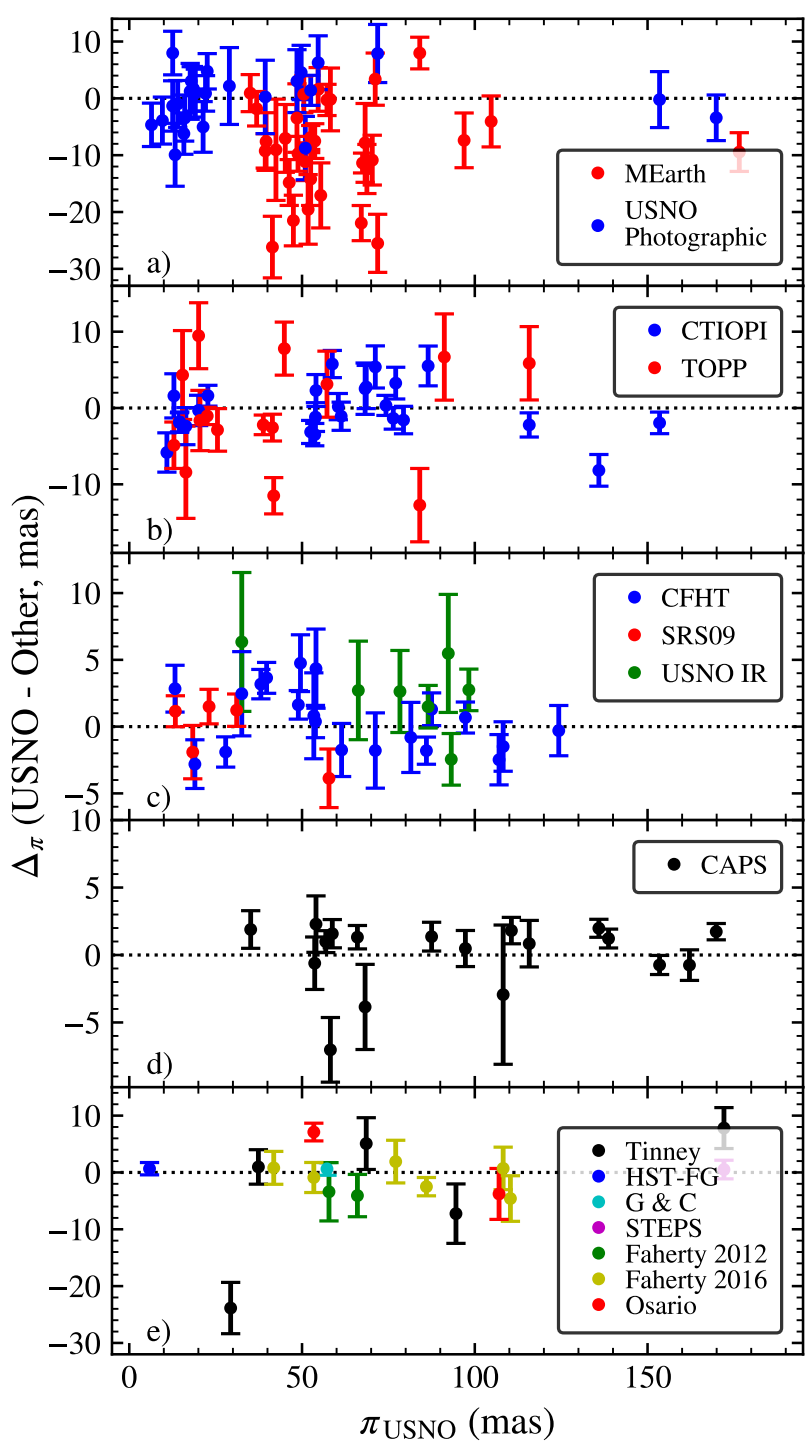

Figure 5. Differences in the parallaxes for stars in common between those presented in this paper and various other programs. Panel (a): MEarth (red) and USNO photographic (blue) parallax programs. Panel (b): RECONS/CTIOPI (blue) and TOPP (red). Panel (c): Infrared parallaxes from Dupuy \& Liu (2012) and Liu et al. (2016, blue), Schilbach et al. (2009, red), and the USNO ASTROCAM parallax program (Vrba et al. 2004, green). Panel (d): CAPS parallax program (Weinberger et al. 2016). Panel (e): Tinney et al. (1995) and Tinney (1996, black), MacConnell et al. (1997, blue), Gatewood \& Coban (2009, cyan), Pravdo \& Shaklan (2009, maroon), Faherty et al. (2012, green), Zapatero Osorio et al. (2014, red), and Faherty et al. (2016, yellow). 
Table 2. Astrometric Comparisons

\begin{tabular}{|c|c|c|c|c|}
\hline $\begin{array}{c}\text { 2MASS J } \\
\text { (1) }\end{array}$ & $\begin{array}{c}\pi_{\mathrm{abs}} \\
(\mathrm{mas}) \\
(2)\end{array}$ & $\begin{array}{c}\mu_{\mathrm{rel}} \\
\left(\operatorname{mas} \mathrm{yr}^{-1}\right) \\
(3)\end{array}$ & $\begin{array}{c}\text { P.A.rel } \\
\text { (deg) } \\
(4)\end{array}$ & $\begin{array}{l}\text { Ref. } \\
\text { (5) }\end{array}$ \\
\hline $00274197+0503417$ & $13.24 \pm 1.56$ & $10.3 \pm 0.3$ & $94.8 \pm 1.5$ & 1 \\
\hline$\cdots$ & $10.4 \pm 0.8$ & $16.7 \pm 1.1$ & $94.8 \pm 3.8$ & 2 \\
\hline $00470038+6803543$ & $81.49 \pm 1.91$ & $435.6 \pm 1.0$ & $116.8 \pm 0.2$ & 1 \\
\hline$\cdots$ & $82.3 \pm 1.8$ & $433.7 \pm 1.2$ & $117.5 \pm 0.2$ & 2 \\
\hline $01144035+4428465$ & $35.02 \pm 0.62$ & $913.9 \pm 0.4$ & $94.7 \pm 0.2$ & 1 \\
\hline$\cdots$ & $34.10 \pm 3.20$ & $887.6 \pm 1.0$ & $94.9 \pm 0.6$ & 3 \\
\hline $01365662+0933473$ & $162.13 \pm 0.57$ & $1237.9 \pm 0.2$ & $91.1 \pm 0.1$ & 1 \\
\hline$\cdots$ & $162.88 \pm 0.98$ & $1222.7 \pm 0.8$ & $90.0 \pm 0.1$ & 4 \\
\hline $01410321+1804502$ & $41.81 \pm 0.73$ & $404.4 \pm 0.3$ & $97.7 \pm 0.1$ & 1 \\
\hline$\cdots$ & $44.06 \pm 2.05$ & $408.1 \pm 0.1$ & $96.9 \pm 0.1$ & 5 \\
\hline$\ldots$ & $41.0 \pm 2.8$ & $412.7 \pm 10.0$ & $96.5 \pm 1.4$ & 6 \\
\hline
\end{tabular}

Note-Table 2 is published in its entirety in machine-readable format. A portion is shown here for guidance regarding its form and content.

References-(1) This paper, Table 1, (2) Liu et al. (2016), (3) Dittmann et al. (2014), (4) Weinberger et al. (2016), (5) Wang et al. (2014), (6) Faherty et al. (2016), (7) Finch \& Zacharias (2016), (8) Tinney et al. (1995), (9) Henry et al. (2006), (10) Gatewood \& Coban (2009), (11) Costa et al. (2005), (12) Jao et al. (2011), (13) Zapatero Osorio et al. (2014), (14) van Altena et al. (1995), Vol. II, (15) Dieterich et al. (2014), (16) Vrba et al. (2004), (17) Dupuy \& Liu (2012), (18) Smart et al. (2010), (19) Khovritchev et al. (2013), (20) Andrei et al. (2011), (21) Smart et al. (2007), (22) Schilbach et al. (2009), (23) Jao et al. (2005), (24) Tinney (1996), (25) Costa et al. (2006), (26) MacConnell et al. (1997), (27) Faherty et al. (2012), (28) Riedel et al. (2010), (29) Gawroński et al. (2017), (30) Pravdo \& Shaklan (2009)

2007, 2010). We have 23 and 15 stars, respectively, in common with these two programs. Both programs employ direct CCD imaging, RECONS/CTIO with $0.9-\mathrm{m}$ and $1.5-\mathrm{m}$ reflectors and TOPP with their 1.05-m scaled-down version of NOFS's Strand Astrometric Reflector. No systematic trends or significant outliers are seen in this comparison.

Parallax determinations carried out at nearinfrared wavelengths include a number of stars in common with our Table 1 sample. Five ultracool subdwarfs in Table 1 of this paper were also observed by Schilbach et al. (2009, hereafter SRS09) at $H$-band using the 3.5-m telescope located in Calar Alto, Spain. Dupuy \& Liu (2012) and Liu et al. (2016) employed the CanadaFrance-Hawaii Telescope (CFHT) to observe a total of 20 of our stars (10 and 10, respectively) either at $J$-band or with a narrow $K_{s}$-band filter. Finally, seven of our stars have preliminary parallaxes from the USNO ASTROCAM program being carried out at NOFS on the Strand Astrometric Reflector (Vrba et al. 2004). These observations were made at either $J$-band or $H$ band. Figure 5, Panel c shows the comparison with our CCD determinations.

Two collaborations, both operating in the southern hemisphere, have commissioned specially designed cameras for astrometrically detecting planets around nearby late-type M, L, and $\mathrm{T}$ dwarfs. A by-product of such observations is the parallax determinations for the target stars. One group, employing a camera with red optimized CCDs on the ESO Very Large Telescope, has presented absolute parallaxes for 20 M8.0V to L2.5V stars (Sahlmann et al. 2014). The precisions of these determinations are unusually good by ground-based standards, with an average uncertainty of \pm 0.10 mas. Unfortunately, none of these stars are in common with ours.

The second collaboration is the Carnegie Astrometric Planet Search (CAPS) program, which employs the $2.5-\mathrm{m}$ du Pont Telescope at the Las Campanas Observatory (Boss et al. 2009). Their camera employs a HAWAII-2RG infrared array with a filter defined bandpass of $\sim 100 \mathrm{~nm}$ FWHM centered at about $865 \mathrm{~nm}-$ optimum for observations of $\mathrm{M}$ dwarfs. Weinberger et al. (2016) have presented absolute parallaxes for 134 of their targets, of which 19 are in common with our Table 1 sample. Except for 2MASS J14162408+1348263, which has the largest parallax error ( \pm 4.99 mas) among their entire 134 targets, their average mean error for the other 18 stars in common with ours is \pm 1.01 mas. Figure 5, Panel d shows the CAPS versus USNO CCD comparison where there is some indication of a small ( $\sim 1.0-1.5$ mas $)$, systematic offset between the two, in the sense that the 
CAPS values are slightly smaller. Such a small difference could possibly be due to differences in arriving at the correction from $\pi_{\text {rel }}$ to $\pi_{\text {abs }}$ for the two programs. That is, the global solutions for CAPS's so-called "zero point offsets" versus the mean photometric parallaxes of the individual reference stars employed by USNO.

Finally, Figure 5, Panel e shows the comparison with 11 additional parallax determinations from 7 different sources: Tinney et al. (1995); Tinney (1996); MacConnell et al. (1997); Gatewood \& Coban (2009); Pravdo \& Shaklan (2009); Faherty et al. (2012); Zapatero Osorio et al. (2014); and Faherty et al. (2016). We note that for the three determinations where the combined errors for the differences with our results are smallest - the HST-FG determination for the distant subdwarf G166-37 (2M1434+2510) by MacConnell et al. (1997); the determination for LHS 269 by Gatewood \& Coban (2009); and the determination for LHS 474 (vB10) by Pravdo \& Shaklan (2009) - there is a small offset of $\sim 1$ mas in the sense that our Table 1 parallaxes are larger. This is similar to what we noted for the CAPS parallax determinations in Figure 5, Panel d. However, the difficulties with observing LHS 474 were noted above, and the small systematics for the other two stars are still less than the combined errors of the comparisons with our results.

\section{PHOTOMETRIC RESULTS}

Photometry in the $V$ and $I$ bands of the Johnson-Kron-Cousins system (Cousins 1976, 1980) was measured for both the astrometric reference stars and and parallax targets in most of the fields using the NOFS $1.0 \mathrm{~m}$ reflector. The secondary photometric standards from Landolt (1983, 1992, 2007, 2013) were employed. Further details regarding our photometric measures can be found in Dahn et al. (2002, Section 3.1). A few fields were observed in the SDSS gri bands (Fukugita et al. 1996) using the NOFS $1.3 \mathrm{~m}$ telescope and transformed to $V$ and $I$ using the relations presented by Ivezić et al. (2007). Where the parallax targets were too faint at $V$-band to be measured at NOFS - and these include many of the earlier $\mathrm{L}$ dwarfs, all of the L dwarfs later than L5, and all four of the T dwarfs $-V-I$ was estimated for stars having spectral types between L0 and L5 from the calibration curve shown in Figure 6. This curve was constructed using stars with measured $V$ band measures from Dieterich et al. (2014) and this study. The third-degree polynomial fit is given by

$$
\begin{aligned}
V-I= & 4.824(0.040)+ \\
& 0.06942(0.024) I_{\mathrm{SpT}}+ \\
& 0.005344(0.0033) I_{\mathrm{SpT}}^{2}+ \\
& 0.007779(0.0015) I_{\mathrm{SpT}}^{3},
\end{aligned}
$$

where $I_{\mathrm{SpT}}$ is an index with zero at spectral type L0, and incremented by numerical spectral subtype. The fit has a standard deviation of 0.20 mag. Thus, an L1.0 star has $I_{\mathrm{SpT}}=1.0$, while an M9.0 star has $I_{\mathrm{SpT}}=-1.0$. This fit should not be extrapolated beyond the boundaries indicated in Figure 6. The $V-I$ values so derived were only employed for the color term in the reduction of actual $I$-band observations.

The VI photometry so obtained was employed for three purposes. First, the $V-I$ color was used to derive DCR corrections for observations taken off of the meridian. However, ever since the TEK2K and EEV24 cameras were commissioned, our policy has been to center each exposure within \pm 15 minutes of meridian crossing. A few frames with exposures centered as much as \pm 30 minutes off the meridian have been retained and used. Hence, DCR is very much a second-order correction for our parallax determinations from these two cameras.

Second, our VI photometry for the reference stars in each of the parallax target fields is used in the selection of which stars actually get employed in the astrometric reductions. The goal here is to avoid using stars that might be nearby, 


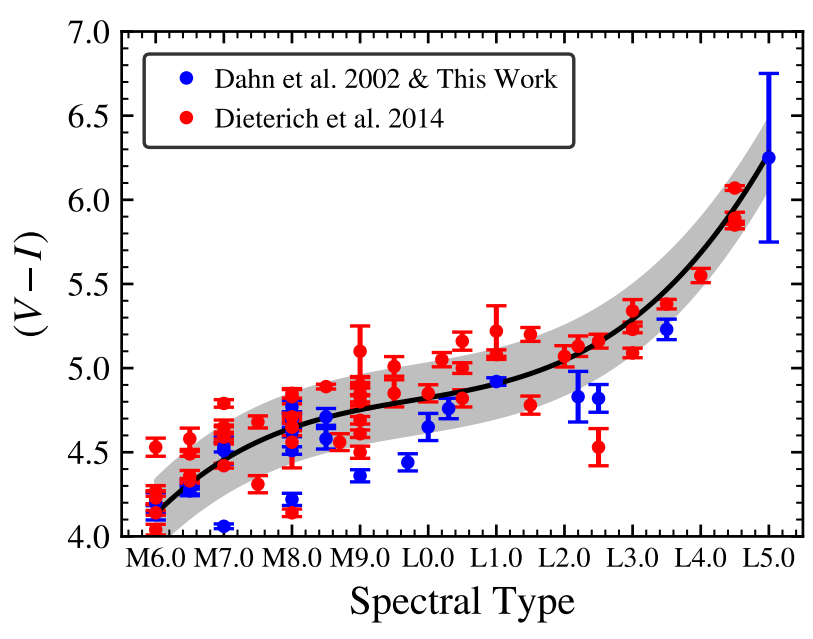

Figure 6. Spectral type versus $V-I$. The fitted curve displays the calibration used to convert spectral type to $V-I$ for stars lacking $V$-band photometry. The gray band represents the standard deviation of the residuals to the fit.

and to that end, whenever possible, red stars are (1) rejected as potentially being nearby dwarfs or (2) only employed if they appear to be distant giants. Figure 7 shows the frequency distribution for the 1992 individual reference stars in all of the TEK2K solutions and for the 517 individual stars employed in all of the TI800 solutions. For the TEK2K fields, the $V-I$ colors peak strongly in the $0.6<V-I<1.0$ range. Since the reference frame stars that we are employing are faint - mostly in the $16<V<18$ range - our calibration for photometric parallax (Harris et al. 2016, Figure 1) places them at distances of generally $1.0-2.0 \mathrm{kpc}$. Reddening/extinction can be important for fields near the galactic plane, and can be handled very satisfactorily as explained in Harris et al. (2016, their Section 3.2).

Third, our photometry for the parallax stars themselves is employed for the color versus absolute magnitude and color versus color diagrams presented in Section 5 below for astrophysical interpretation. The parallax stars are mostly at distances less than 100 pc, and hence, within the so-called "Local Bubble" (Fitzger-

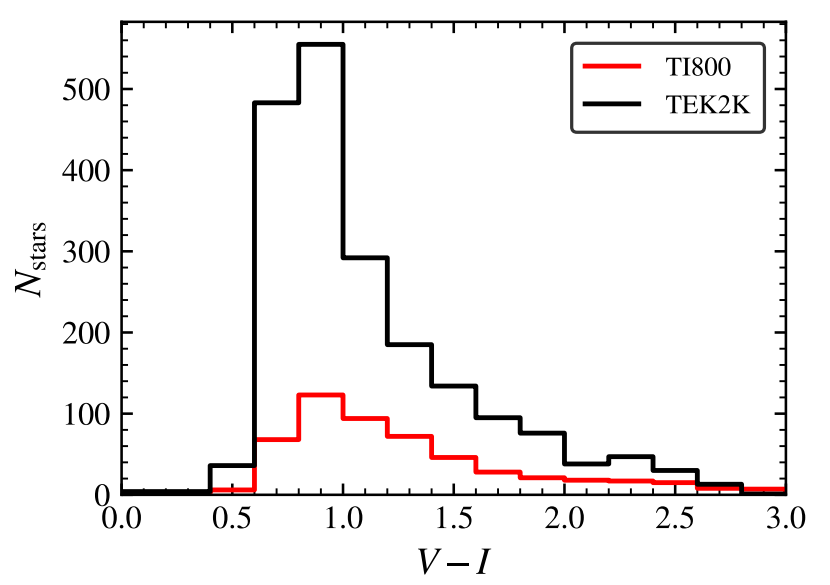

Figure 7. Distribution of reference stars in $V-I$ color, shown separately for the TI800 and TEK2K programs.

ald 1968; Frisch et al. 2011). This a volume around the sun wherein, even in the galactic plane, extinction by interstellar dust is minimal, and which extends hundreds of parsec in the directions toward the galactic poles. However, in a few directions - such as for our three fields toward the Hyades cluster - it can still be an issue.

Table 3 presents our NOFS photometric results for each of the parallax stars in Table 1 . Column (1) is the same as in Table 2. Columns (2) and (3) are the NOFS $V$ and $I$ measures along with their formal uncertainties, all rounded to the nearest $0.01 \mathrm{mag}$. The results quoted for $V$ and $I$ include measures previously reported in Monet et al. (1992) and in Dahn et al. (2002). Hence, the Table 3 values supersede the earlier ones. Where the star was too faint to be measured at $V$-band, and the spectrally determined $V-I$ was employed for the color term in the $I$-band reductions (see above), the quoted uncertainty for $I$ has been adjusted upward appropriately. And since Figure 6 could neither be calibrated for L dwarfs/subdwarfs later than L5 nor for T dwarfs, further uncertainties in the reported $I$ magnitudes are flagged with a colon. 
Table 3. Photometric Results

\begin{tabular}{|c|c|c|c|c|c|c|c|}
\hline $\begin{array}{c}\text { Name }^{\mathrm{a}} \\
(1)\end{array}$ & $\begin{array}{l}V \\
(2)\end{array}$ & $\begin{array}{c}I \\
(3)\end{array}$ & $\begin{array}{c}J \\
(4)\end{array}$ & $\begin{array}{l}H \\
(5)\end{array}$ & $\begin{array}{r}K_{S} \\
(6)\end{array}$ & $\begin{array}{l}\mathrm{SpT} \\
(7)\end{array}$ & $\begin{array}{c}\text { Type }^{b} \\
(8)\end{array}$ \\
\hline $00110078+0420245$ & $17.89 \pm 0.02$ & $15.57 \pm 0.02$ & $14.34 \pm 0.03$ & $13.81 \pm 0.05$ & $13.76 \pm 0.04$ & usdM4.5 & 5 \\
\hline $00192745+0450297$ & $17.25 \pm 0.02$ & $13.75 \pm 0.02$ & $11.98 \pm 0.03$ & $11.40 \pm 0.03$ & $11.09 \pm 0.02$ & M5.5V & 1 \\
\hline $00274197+0503417$ & $\ldots$ & $19.79 \pm 0.10$ & $16.19 \pm 0.09$ & $15.29 \pm 0.10$ & $14.96 \pm 0.12$ & M9.5pecV & 1 \\
\hline $00312326+0936169$ & $16.56 \pm 0.02$ & $14.72 \pm 0.02$ & $13.64 \pm 0.02$ & $13.10 \pm 0.03$ & $12.91 \pm 0.03$ & $\cdots$ & $3: \mathrm{v}$ \\
\hline $00350768+7627544$ & $17.78 \pm 0.02$ & $13.88 \pm 0.02$ & $11.65 \pm 0.02$ & $11.06 \pm 0.03$ & $10.70 \pm 0.03$ & . & 1 \\
\hline $00361617+1821104$ & $21.34 \pm 0.06$ & $16.11 \pm 0.01$ & $12.47 \pm 0.03$ & $11.59 \pm 0.03$ & $11.06 \pm 0.02$ & $\mathrm{~L} 3.5$ & 6 \\
\hline $00470038+6803543$ & $\cdots$ & $19.77 \pm 0.12:$ & $15.60 \pm 0.07$ & $13.97 \pm 0.04$ & $13.05 \pm 0.03$ & L7pec & 6 \\
\hline $00475502+4744342$ & $16.00 \pm 0.05$ & $13.20 \pm 0.05$ & $11.68 \pm 0.02$ & $11.17 \pm 0.02$ & $10.91 \pm 0.02$ & $\cdots$ & $2: 1$ \\
\hline $00510351-1411047$ & $19.82 \pm 0.04$ & $16.12 \pm 0.03$ & $14.09 \pm 0.03$ & $13.53 \pm 0.02$ & $13.32 \pm 0.03$ & $\cdots$ & $3: \mathrm{v}$ \\
\hline $00554418+2506235$ & $18.94 \pm 0.03$ & $15.76 \pm 0.02$ & $14.26 \pm 0.03$ & $13.78 \pm 0.03$ & $13.57 \pm 0.04$ & sdM6.5 & 3:vs \\
\hline
\end{tabular}

Note-Table 3 is published in its entirety in machine-readable format. A portion is shown here for guidance regarding its form and content.

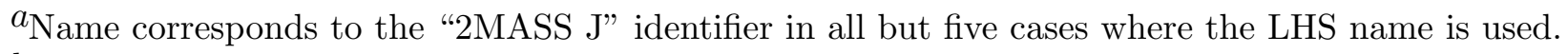

${ }^{b}$ Our adopted types are as follows: (1) K or M Dwarf; (2) an uncertain subdwarf with $150 \leq \mathrm{V}_{\tan }<180$ $\mathrm{km} \mathrm{s}^{-1}$ and/or showing marginal subluminosity in one or more of the diagrams presented in Section 5 ; (3) K or M subdwarf (but not extreme nor ultra); (4) exteme M subdwarf (esdM); (5) ultra M subdwarf (usdM); (6) L or T dwarf (d); (7) L subdwarf (sdL); (8) extreme L subdwarf (esdL); (9) ultra L subdwarf (usdL). Type assignments for $3-9$ are based on observed spectroscopic classifications found in the literature. For types 2 and 3, a following letter indicates the supporting evidence for our typing decision where: $\mathrm{v}=\mathrm{V}_{\tan } \geq$ $180 \mathrm{~km} \mathrm{~s}^{-1} ; \mathrm{s}=$ spectral type; and $\mathrm{l}=$ significant subluminosity in one or more of color-absolute magnitude diagrams presented in Section 5.

Columns (4) - (6) in Table 3 give the 2MASS $J H K_{s}$ magnitudes extracted from the Point Source Catalog (Skrutskie et al. 2006) and again rounded to the nearest 0.01 mag.

Column (7) of Table 3 gives, where available, representable spectral types extracted from the literature. Of the total 309 stars, spectral typings were located for $245(\sim 80 \%)$. (We do not consider the Luyten color classes - such as k, $\mathrm{k}-\mathrm{m}, \mathrm{m}$ and $\mathrm{m}+-$ as legitimate spectral types since they merely represent broadband photographically determined colors with significant measurement uncertainties.) The spectral types that we adopt are not on any homogeneous sys- tem, and we offer no attempt to do so. Of the 245 stars for which we quote types, the majority $(\sim 75 \%)$ are $\mathrm{M}$ stars - either dwarfs or subdwarfs. The remaining include (disregarding several faint, unresolved secondary companions in binary systems) 6 late $\mathrm{K}$ dwarfs or subdwarfs, $43 \mathrm{~L}$ dwarfs, $6 \mathrm{~L}$ subdwarfs, and $4 \mathrm{~T}$ dwarfs.

For the $\mathrm{M}$ dwarfs, we give priority to typings on the system set up by Kirkpatrick et al. (1991). As for M subdwarfs, Jao et al. (2008) have presented an excellent summary of the evolution of K-M subdwarf spectral typings. Using multichannel spectrophotometric observations of a large sample of cool (late-type) proper 
motion stars, Ake \& Greenstein (1980) identified four stars - LHS 453, LHS 3382, G 717 and LHS 498 - for which the metallicity indicators $\mathrm{TiO}, \mathrm{CaH}, \mathrm{MgH}$, and $\mathrm{CaI} \lambda 4227 \AA$ indicated extreme metal deficiency. Hartwick et al. (1984) used photometric and spectroscopic observations to establish narrowband indices measuring the relative strengths of the $\mathrm{TiO}$ and $\mathrm{CaH}$ features in the $\lambda \lambda 6800-7600 \AA$ wavelength range. Gizis (1997) and Gizis \& Reid (1997a,b) followed with a similar scheme that employs three indices for measuring the strength of $\mathrm{CaH}$ along with a single index measuring the strength of $\mathrm{TiO}$ in the $\lambda \lambda 6200-7400 \AA$ window. This enabled $\mathrm{M}$ dwarfs to be identified as either dwarfs, subdwarfs (sdM) or extreme subdwarfs (esdM). As originally defined, this system only covered earlier M subdwarfs, down to $\sim$ M5. Among the very coolest subdwarfs, LHS 377 was set to define the type sdM7.0 (Gizis \& Harvin 2006) since it was the only subdwarf later than $\sim \operatorname{sdM} 5$ known at that time. Extension to cooler subdwarf M stars was carried out by Schweitzer et al. (1999), Lépine et al. (2003), Lépine et al. (2004), and Scholz et al. (2004). Lépine et al. (2007) then proposed a revision to this classification scheme by introducing an additional parameter that measures the strength of the $\mathrm{TiO}$ compared with a similar star possessing solar metallicity. This resulted in establishing an additional subclass of "ultrasubdwarf" corresponding to subdwarfs with the lowest metal content. Lépine et al. (2007, Table 2) presented a listing of classification standard stars for all subclasses sd, esd, and usd. In our Table 1 we present parallax determinations for 11 of these stars - LHS 228 (sdM2.0); LSR 1425+7102 (sdM8.0); LHS 360 (esdM0.0); LHS 2045 (esdM5.0); LEHPM 259 (esdM8.5); LHS 2843 (usdM0.0); LHS 325 (usdM3.5); LHS 1032 (usdM4.5); LHS 2500 (usdM5.0); LSPM J0822+1700 (usdM7.5); and 2MASS J12270506-0447207 (usdM8.5).
Jao et al. (2008) pointed out that this current system is somewhat deficient in that it utilizes a rather limited region of the spectrum at low resolution $(\lambda / \Delta \lambda \sim 3,000)$ and does not adequately link the low-metallicity subdwarfs to their main-sequence counterparts with respect to temperature and surface gravity. Woolf et al. (2009) argue that the gravity will only be an issue for young stars that have not yet fully settled onto the main sequence. Using high-dispersion spectra covering $\lambda \lambda 6200-9800 \AA$ $(\lambda / \Delta \lambda \sim 33,000$, resolution $\sim 3.1 \AA)$ to determine $\mathrm{Fe}$ and $\mathrm{Ti}$ abundances, they demonstrate that the low-resolution $\mathrm{CaH}$ and $\mathrm{TiO}$ indices do measure valid metallicity discrimination among the sd, esd, and usd subclasses for stars with $3500 \mathrm{~K}<T_{\text {eff }}<4000 \mathrm{~K}$ and with $-1.5<[\mathrm{Fe} / \mathrm{H}]<+0.05$. Cooler subdwarfs (e.g., the ultracool ones later than M7) are too faint and are not currently observable with similar high-resolution spectroscopy.

Zhang et al. (2017b) have now extended the sd, esd, and usd subclasses to L-type stars. Optical spectra in the $\lambda \lambda 7300-8800 \AA$ wavelength region were employed to tie the L-subdwarfs to the L-dwarf spectral standard stars (Burgasser et al. 2007; Kirkpatrick et al. 1999, 2010). Although molecular bands at optical wavelengths (e.g., $\mathrm{CaH}, \mathrm{TiO}, \mathrm{VO}$, and $\mathrm{FeH}$ ) remain important in establishing the L-subdwarf subclasses, near-infrared spectral behavior out to $\sim 2.5$ microns become very important. The defining characteristics for the L-subdwarf subclasses are outlined in Table 3 of Zhang et al. (2017b).

Three of the stars in our sample have now been reclassified into this new system. SSSPM J1013-1356 was previously classified as sdM9.5 but now is classified as usdL0,

2MASS J1626034+3925190 was formerly classified as sdL4 but now becomes usdL4, and LSPM J1826+3014 is now typed sdL0 rather than d/sdM8.5. Further modifications to this 
system can be expected as the sample of Lsubdwarfs grow.

The final column in Table 3 indicates the star "type" that we adopt for each target based on the aggregate astrometric, photometric, spectroscopic, and kinematic information available to us. This "type" is meant to indicate whether each star is most likely to be a dwarf, subdwarf, extreme subdwarf, or ultrasubdwarf and will be employed for to present the diagrams in Section 5.

\section{SAMPLE COLOR-ABSOLUTE MAGNITUDE AND COLOR-COLOR DIAGRAMS}

Tables 1 and 3 above provide the basis for a large number of empirical color-absolute magnitude and color-color diagrams. Only a sample will be presented here. Figure 8 shows $M_{V}$ versus $V-I$ (left panel) and $M_{V}$ versus $V-K_{s}$ (right panel) for the entire sample with observed $V I$ photometry in Table 3. The filled black circles are $\mathrm{K}$ and $\mathrm{M}$ dwarfs. The filled blue circles are M subdwarfs (sdM, esdM, and usdM types). The green symbols are all L dwarfs, where the solid circles are dL types, the solid square is the sdL star 2MASS J14162408+1348263, and the filled triangles are the two usdL stars in our sample, SSSPM J1013-1356 and 2MASS $\mathrm{J} 1626034+3925190$. The red line is the nominal dwarf main sequence as presented in Harris et al. (2016). Here (and in all subsequent figures), formal $\pm 1 \sigma$ error bars are included for all data points plotted.

One general feature of note seen in $M_{V}$ versus $V-I$ is a hook back to bluer colors for the latest M-dwarfs. This is recognized to be an effect of the change in spectral energy distribution (primarily changes in the strength of various prominent $\mathrm{TiO}$ absorption bands) across the $V$ and $I$ bandpasses as the sequence progresses to cooler temperatures. No such deviation is seen in the $M_{V}$ vs $V-K_{s}$ diagram.
A few individual stars warrant special attention as obvious outliers. SSSPM J1013-1356 has recently been reclassified to spectral type usdL0 (Zhang et al. 2017b) versus the earlier classification of sdM9.5 originally announced by Scholz et al. (2004). This star's location in Figure 8 is somewhat difficult to understand as an L star since it is roughly an order of magnitude more luminous than any of the other $\mathrm{L}$ dwarfs and subdwarfs in the plot. Clearly, the problem is not with the astrometry. Our Table 1 parallax is in very good agreement with the independent determination by Schilbach et al. (2009). Likewise, the photometry is believed to be reliable to the precision reported in Table 3. A spectral classification of very late esdM or usdM would clearly be more compitable with our results.

2MASS J16262034+3925190 was first announced as sdL4 by Burgasser (2004). Lodieu et al. (2015) observed an upper limit of $90 \mathrm{~m} \AA$

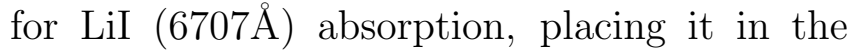
Li-burning regime. They also measured an improved $V_{\text {rad }}=239 \pm 12 \mathrm{~km} \mathrm{~s}^{-1}$ which, along with our $V_{\tan }=212 \mathrm{~km} \mathrm{~s}^{-1}$, strongly supports halo candidacy. This star stands out in the $M_{V}$ vs $V-K_{s}$ diagram but does not stand out in $M_{V}$ vs $V-I$. As we noted above in Section 3.3, the residuals from our parallax astrometry suggest a low-amplitude perturbation.

The three dwarfs located in the direction of the Hyades cluster, but apparently well beyond it, - 2MASS J04193967+1433329, 2MASS J04223075+1526310, and

2MASS J04325119+1730092 - fall significantly above the dwarf sequence, as do two of the three moving group candidates (2MASS J04435686+3723033 and 2MASS J21374019+0137137). As noted in Sec. 3 above, images of 2MASS J21374019+0137137 taken in very good seeing suggest a partially resolved binary system. Also falling above the dwarf sequence are (1) 2MASS J03350208+2342356, an M8.5V star with de- 


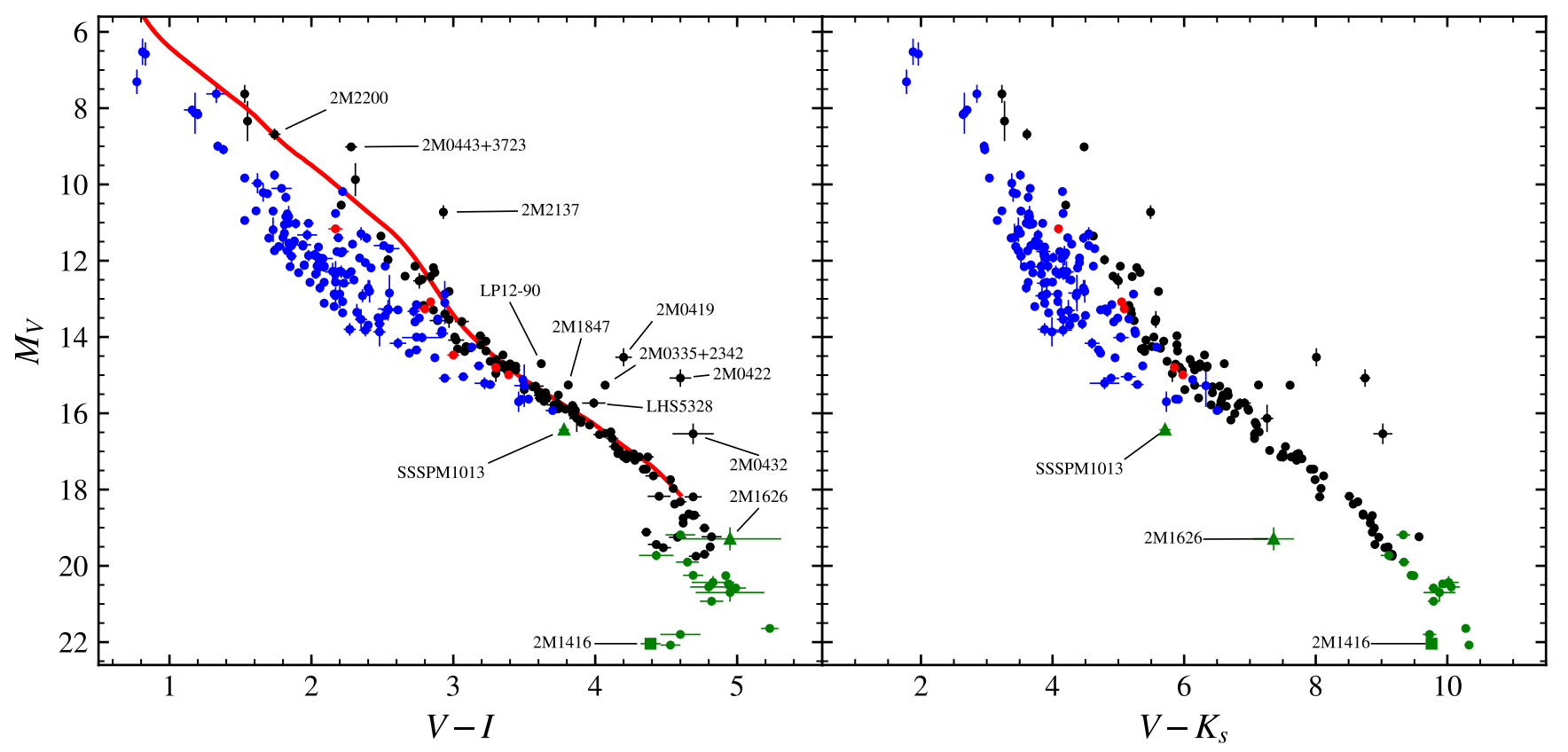

Figure 8. $M_{V}$ versus $V-I$ (left panel) and $V-K_{s}$ (right panel) for all stars with $V I$ photometry. Symbols are: $\mathrm{K}$ and $\mathrm{M}$ dwarfs (black circles), $\mathrm{M}$ subdwarfs (blue circles), dL (green circles), sdL (green squares), usdL (green triangles), unknown dwarf/subdwarf status (red circles). The red line is the nominal dwarf main sequence from Harris et al. (2016).

tected LiI absorption, consistent with pre-mainsequence status, and (2) stars LP12-90, 2MASS J18470342+5522433, and LHS 5328 which appear to be unresolved binaries with nearly equal-luminosity components.

Figure 9 shows an expanded view of the $\mathrm{K}$ and M subdwarf region of the $M_{V}$ vs $V-I$ plane. Again, $\mathrm{K}$ and $\mathrm{M}$ dwarfs are represented by solid black circles, while the sdK and sdM stars are shown as filled blue circles. The solid red circles are stars for which the dwarf versus subdwarf status is uncertain. The open blue circles are esdM stars and the solid filled blue triangles are usdM stars. Individual stars labeled include the 11 spectral classification standards from Lépine et al. (2007, Table 2) and LHS 377, the sdM7.0 standard designated by Gizis \& Harvin (2006) but reclassified as esdM7.0 by Zhang et al. (2017b). The solid blue lines are model locii for metal-poor stars from Baraffe et al. (1997, 1998). Also labeled is LHS 507 which was independently identified to be a late subdwarf
K star by Bessell \& Wickramasinghe (1979) using broadband photometry and by Liebert et al. (1979) using low-resolution spectroscopy. The location of LHS 507 in Figure 9 indicates $[\mathrm{M} / \mathrm{H}]=-2.0$ such that modern spectroscopy should confirm it to be of usdK type. The location of SSSPM J1013-1356 is represented by a solid green triangle.

Comparing our Figure 9 observational results with Baraffe et al. (1997, 1998) models shows no evidence for stars more metal poor than $[\mathrm{M} / \mathrm{H}]=-2.0$. Further spectroscopic observations of many of the stars only identified as subdwarfs based on location in our diagrams and/or large measured $V_{\text {tan }}$ values (e.g., LHS 335, LHS 1894, LHS 1953, LHS 2929, LHS 3207) should establish additional esdM- and usdM-type stars.

Figure 10 shows our sample in $M_{K_{s}}$ versus $I-K_{s}$ (left panel) and $M_{K_{s}}$ versus $J-K_{s}$ (right panel), focusing on the $\mathrm{L}$ dwarfs. Here, the $\mathrm{dL}$ stars are represented by filled green circles, the sdL stars by open green circles, the usdL stars 


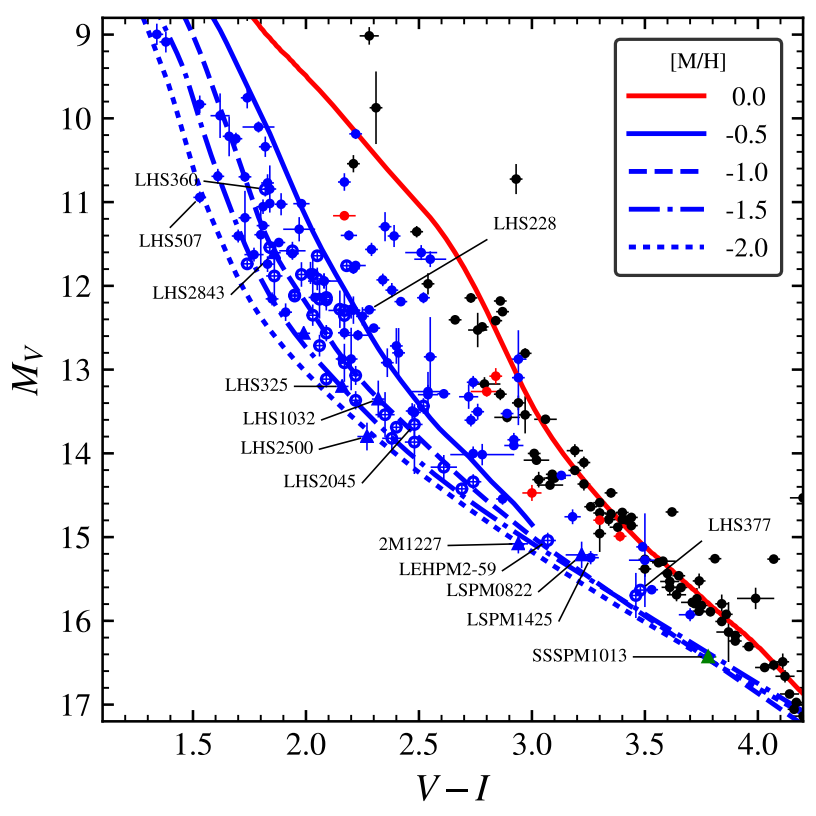

Figure 9. $M_{V}$ versus $V-I$ for all stars with $V I$ photometry, zoomed in on the $\mathrm{K}$ and $\mathrm{M}$ subdwarf region. Symbols are: $\mathrm{K}$ and $\mathrm{M}$ dwarfs (filled black circles), sdK and sdM (filled blue circles), dwarf/subdwarf status unknown (filled red circles), esdM (open blue circles), usdM (filled blue triangles), SSSPM J1013-1356 (green triangle). The red line is the nominal dwarf main sequence from Harris et al. (2016). Blue lines are model locii for metal poor stars from Baraffe et al. (1997, 1998).

by open green triangles, and the two $\mathrm{T}$ dwarfs with I-band photometry (2MASS J125453930122474 and 2MASS J05591914-1404488) by open green squares. The single esdL star in our sample (SSSPM J1444-2019) lacks I-band photometry.

Figure 11 presents an expanded view of the $\mathrm{K}$ and $\mathrm{M}$ dwarf region of the $M_{K_{s}}$ versus $I-K_{s}$ plane. Symbols employed are as described for Figure 9. Additionally, sdL stars are represented by open green circles and usdL stars by open green triangles. Again, there seems to be many sdM stars awaiting confirmation as esdM and usdM types. As in Figure 9, there is scant evidence for stars with $[\mathrm{M} / \mathrm{H}]<-2.0$.

These color-magnitude diagrams using IR colors can provide an excellent diagnostic for metallicity, and thus for subluminosity and for stellar population membership. They are independent of spectral classification, and so will be useful in interpreting the results of Gaia parallaxes for many late-type dwarfs soon to be available (see Section 6 below). Early versions of these diagrams were shown by Dahn \& Harris (2015).

Figure 12 shows the $I-J$ versus $J-K_{s}$ colorcolor diagram. The hook toward bluer $J-K$ colors at the top of the diagram shows the onset of methane absorption in the $K$-band for $\mathrm{T}$ dwarfs and the coolest $\mathrm{L}$ dwarfs. The other outliers toward blue $J-K$ colors are the extreme subdwarfs identified in previous figures. Zhang et al. (2017b) recently employed an equivalent version of this diagram (using $J-K_{s}$ versus $i-J$, where $i$ is the SDSS magnitude) to identify six new L-type subdwarfs.

\section{DISCUSSION}

The parallax determinations reported in the present paper are intended to sample (to the extent practical) a full range of metal-poor verylate-type stars. The goals were to document the spread in luminosity among these stars (cf. Figure 8), to utilize color-magnitude diagrams to classify a star's metallicity and population (cf. Figure 9), and to facilitate understanding of the physical causes affecting their colors and spectral types. Spectroscopic classification for late-M and $\mathrm{L}$ subdwarfs is still being developed. For example, Jao et al. (2008) suggest that the wavelength regions using the $\mathrm{CaH}$ and $\mathrm{TiO}$ bands to establish the sd, esd, and usd metallicity subclasses are also sensitive to surface gravity and do not accurately reflect metallicity alone. Thus, more work is needed in spectrophotometric modeling for a comprehensive understanding. Here, we suggest an alternative path toward classification independent of spectral type; based on color and parallax measurements, it can be applied to the large sample 


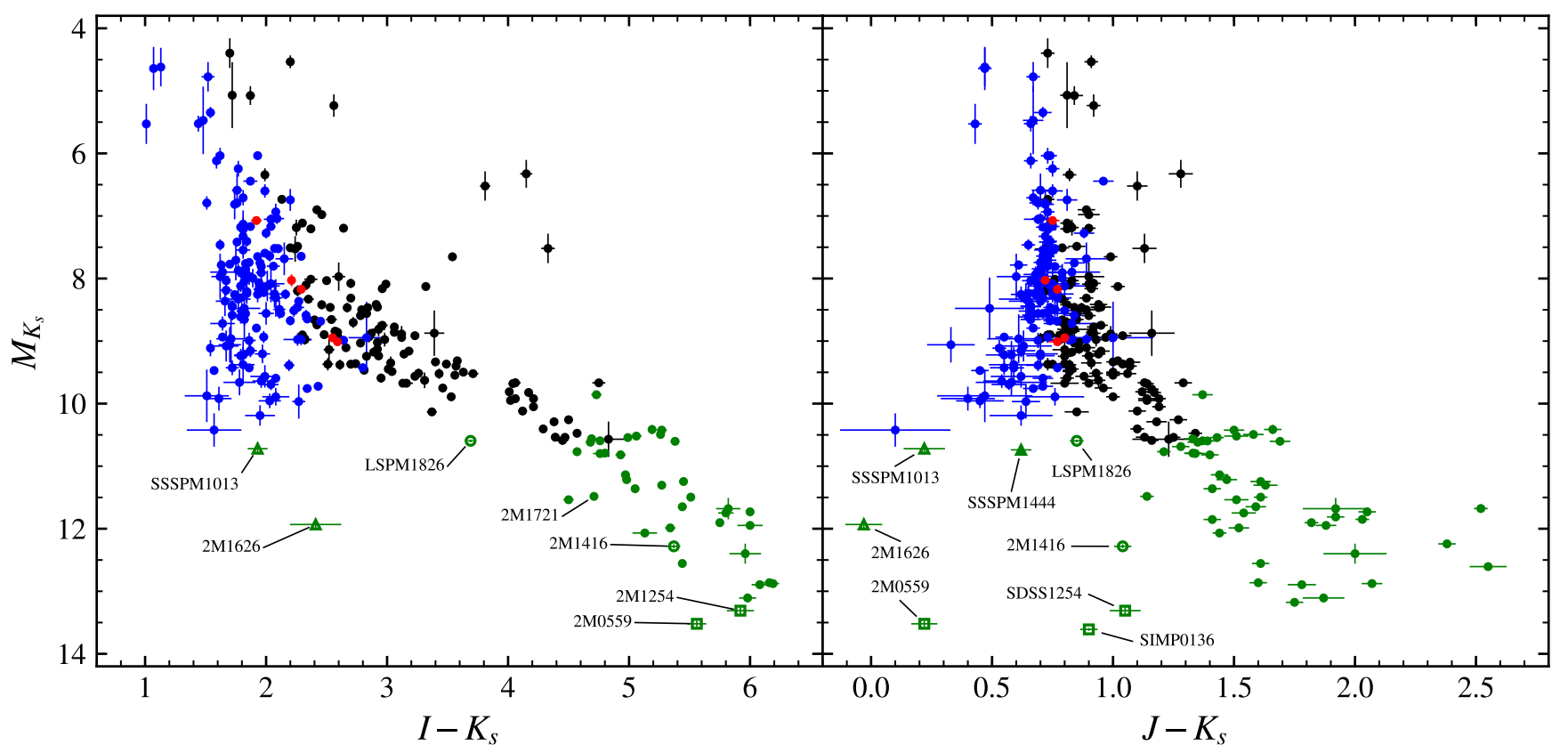

Figure 10. $M_{K_{s}}$ versus $I-K_{s}$ (left panel) and $J-K_{s}$ (right panel) for our sample, zoomed in on the L dwarfs. Symbols are: K and M dwarfs (filled black circles), sdK and sdM (filled blue circles), dL (filled green circles), sdL (open green circles), usdL (open green triangles), T dwarfs (open green squares).

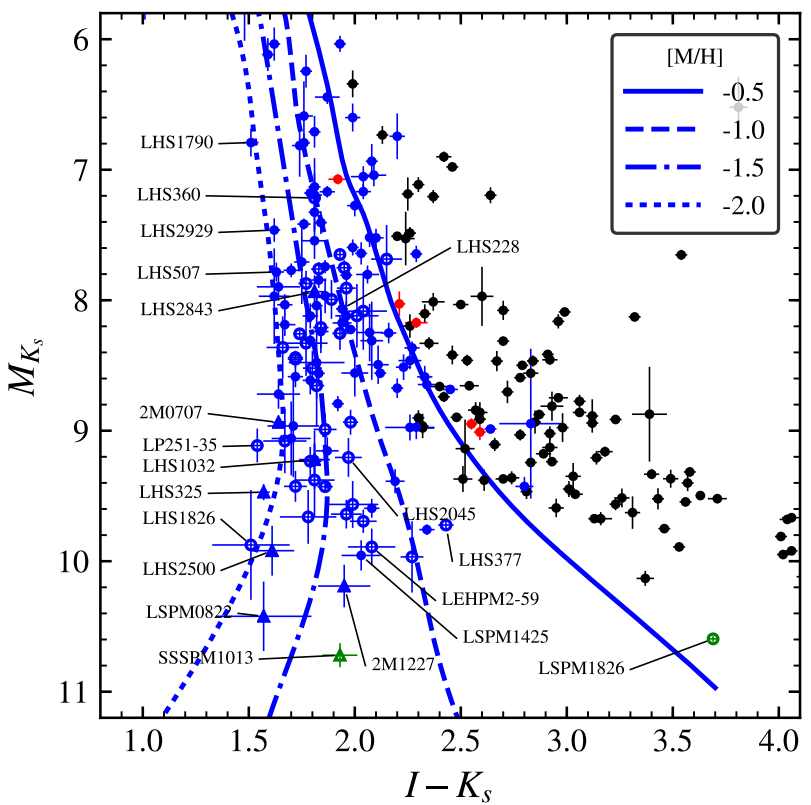

Figure 11. $M_{K_{s}}$ versus $I-K_{s}$, zoomed in on the $\mathrm{K}$ and $\mathrm{M}$ dwarf region. Symbols are the same as for Figure 9, with the following additional symbols: sdL (open green circles), usdL (open green triangles).

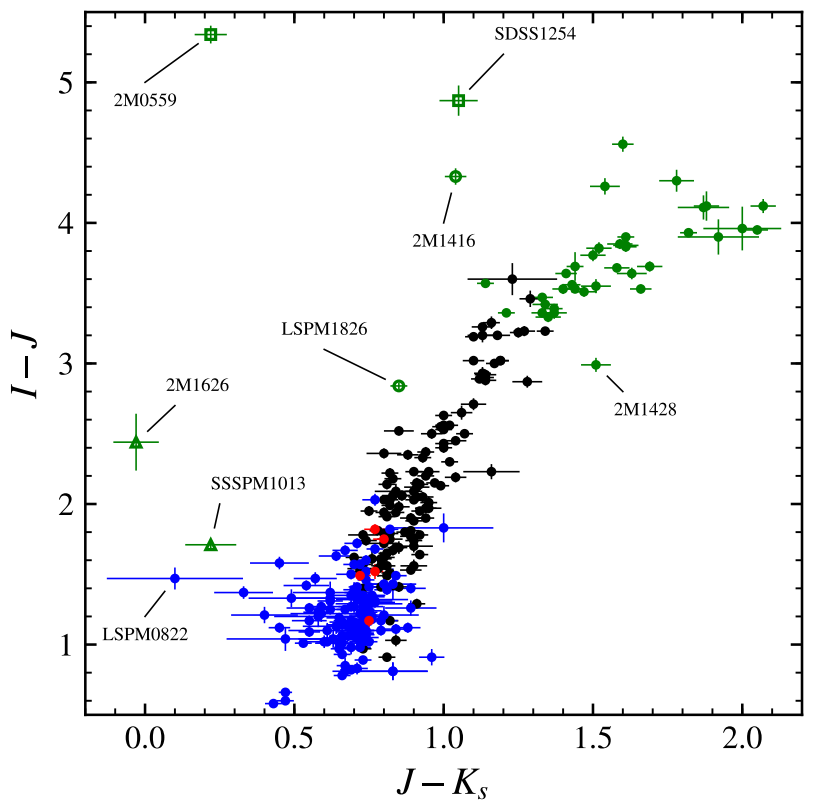

Figure 12. $I-J$ versus $J-K_{s}$ color-color diagram. Symbols are the same as for Figure 10

of objects that will soon have these data from Gaia and LSST.

A decade ago, there were only sixteen spectroscopically confirmed ultracool subdwarfs 
(Burgasser et al. 2007). Of these, all but two $(2 \mathrm{M} 0937+2931$ and $2 \mathrm{M} 0532+8246)$ had spectral types between M7 and L4. Shortly thereafter Lépine \& Scholz (2008) announced the identification of 23 additional ultracool M-type subdwarfs from SDSS DR6 spectra. More recently, Lodieu et al. (2017) presented M-type subdwarf identifications from their cross-matching of 2MASS, SDSS, and UKIDSS databases. Included are a total of 27 M-type ultracool subdwarfs (types 7.0 or later) plus three early-type sdL stars. Since then a considerable number of additions have been identified, including SDSS J010448.46+153501.8 which Zhang et al. (2017a) claim as the "the most metal-poor substellar object" currently known with $[\mathrm{Fe} / \mathrm{H}]=-2.4 \pm 0.2$ and a spectral type usdL1.5. These additions bring the number of known ultracool subdwarfs to around 60 .

Gaia, a cornerstone mission in the science program of the European Space Agency (ESA; Gaia Collaboration et al. 2016a,b), will measure parallaxes for large numbers of $\mathrm{M}$ dwarfs and subdwarfs. For a Gaia apparent magnitude of $G=20$, the sky-averaged, end-of-mission parallax error is projected to be \pm 0.3 mas (de Bruijne 2014). Employing the $G$ versus $V, V-I$ cubic polynomial relation from Jordi et al. (2010, Table 6) - updated with the coefficients from de Bruijne (2014) - we estimate that for a verylate M-dwarf (typically with $M_{V} \sim 19$ and $V-I \sim 4.7$; cf. Figure 8), Gaia will reach to $V \sim 23.2$, corresponding to a distance of roughly 70 pc. Adopting the local luminosity function from Just et al. (2015), Gaia should measure parallaxes for $\sim 1700 M_{V} \sim 19$ dwarfs, all to an accuracy of $2 \%$ or better. Similar estimates for the earlier-type (M7 and M8) ultracool dwarfs indicate that Gaia will observe them, on average, to distances of $110 \mathrm{pc}$ and $90 \mathrm{pc}$, respectively, leading to measurements of roughly 25000 of the M7 \& M8 dwarfs. Furthermore, the actual Gaia limiting magnitude will be $G \sim 20.7$, resulting in measurements for additional stars, with somewhat increased parallax errors.

Thus, Gaia will provide an orders-of-magnitude greater sample size for $M$ dwarfs, and, by extension, an increased sample of early-L dwarfs. For mid-L and cooler dwarfs, the rapidly fading absolute magnitude in the Gaia G-band will severely limit the distance at which they will be observed. Similarly, for ultracool subdwarfs, the Gaia limiting magnitude and their low space density will limit the sample size. Here, the Large Synoptic Survey Telescope (LSST; Ivezic et al. 2008) should extend samples of ultracool dwarfs to apparent magnitudes much fainter than $G=21$. Commencing observations around 2020, LSST will measure parallaxes with an accuracy that will depend on several issues still to be finalized, especially the cadence of the observations. (LSST astrometry will require correction for differential color refraction and correction from relative to absolute parallax values.) Several participants at the 2014 Torino workshop (cf. de Bruijne 2014) noted that the projected error of the LSST parallaxes will be $\approx \pm 0.4$ mas for stars with SDSS $r$-band magnitudes over the range from $\sim 16.0$ to $\sim 20.0$. Hence, there should be an important overlap with Gaia's absolute parallax determination.

We sincerely thank Bill van Altena for his continued support of the NOFS parallax program, especially during the late phases of the photographic era and the transition to CCD detectors. Special thanks go to Jim Liebert and Pat Boeshaar for communicating results from their spectroscopic investigations to us, allowing our astrometric observations to commence as soon as possible. Likewise, we thank the members of the 2MASS Rare Objects Team - especially Davy Kirkpatrick, Neill Reid, and Kelle Cruz - for timely alerting us in a timely matter about interesting targets. We further thank 
the anonymous referee for many helpful suggestions. This research has made use of the SIMBAD database, operated at CDS, Strasburg, France. We greatly appreciate the dedicated efforts. Special thanks are extended to the Engineering Team here at NOFS - Mike DiVittorio, Al Rhodes, Mike Schultheis, Alan Bird, and Steve Sell - for maintaining the Strand Astrometric Reflector in top condition.

This publication makes use of data products from the Two Micron All Sky Survey, which is a joint project of the University of Massachusetts and the Infrared Processing and Analysis Cen-
ter/California Institute of Technology, funded by the National Aeronautics and Space Administration and the National Science Foundation.

This work has made use of data from the European Space Agency (ESA) mission Gaia (https://www.cosmos.esa.int/gaia), processed by the Gaia Data Processing and Analysis Consortium (DPAC, https://www. cosmos . esa.int/web/gaia/dpac/consortium). Funding for the DPAC has been provided by national institutions, in particular the institutions participating in the Gaia Multilateral Agreement.

\section{REFERENCES}

Ake, T. B., \& Greenstein, J. L. 1980, ApJ, 240, 859

Andrei, A. H., Smart, R. L., Penna, J. L., et al. 2011, AJ, 141, 54

Bakos, G. Á., Sahu, K. C., \& Németh, P. 2002, ApJS, 141, 187

Baraffe, I., Chabrier, G., Allard, F., \& Hauschildt, P. H. 1997, A\&A, 327, 1054

-. 1998, A\&A, 337, 403

Bean, J. L., Seifahrt, A., Hartman, H., et al. 2010, ApJL, 711, L19

Bertin, E., \& Arnouts, S. 1996, A\&AS, 117, 393

Bessell, M. S., \& Wickramasinghe, D. T. 1979, ApJ, 227, 232

Blake, C. H., Charbonneau, D., \& White, R. J. 2010, ApJ, 723, 684

Boss, A. P., Weinberger, A. J., Anglada-Escudé, G., et al. 2009, PASP, 121, 1218

Bouy, H., Brandner, W., Martín, E. L., et al. 2003, AJ, 126, 1526

Burgasser, A. J. 2004, ApJL, 614, L73

Burgasser, A. J., Cruz, K. L., \& Kirkpatrick, J. D. 2007, ApJ, 657, 494

Burningham, B., Cardoso, C. V., Smith, L., et al. 2013, MNRAS, 433, 457

Costa, E., Méndez, R. A., Jao, W.-C., et al. 2005, AJ, 130, 337

—. 2006, AJ, 132, 1234

Cousins, A. W. J. 1976, MmRAS, 81, 25

- 1980, South African Astronomical Observatory Circular, 1, 234
Dahn, C. C., \& Harris, H. C. 2015, in Cambridge Workshop on Cool Stars, Stellar Systems, and the Sun, Vol. 18, 18th Cambridge Workshop on Cool Stars, Stellar Systems, and the Sun, ed. G. T. van Belle \& H. C. Harris, 959-966

Dahn, C. C., Harris, H. C., Vrba, F. J., et al. 2002, AJ, 124, 1170

de Bruijne, J. H. J. 2014, Mem. Soc. Astron. Italiana, 85, 631

Dieterich, S. B., Henry, T. J., Jao, W.-C., et al. 2014, AJ, 147, 94

Dittmann, J. A., Irwin, J. M., Charbonneau, D., \& Berta-Thompson, Z. K. 2014, ApJ, 784, 156

Dupuy, T. J., \& Liu, M. C. 2012, ApJS, 201, 19

—. 2017, ArXiv e-prints, arXiv:1703.05775

Dupuy, T. J., Liu, M. C., \& Ireland, M. J. 2009, ApJ, 699, 168

Faherty, J. K., Burgasser, A. J., Bochanski, J. J., et al. 2011, AJ, 141, 71

Faherty, J. K., Burgasser, A. J., Cruz, K. L., et al. 2009, AJ, 137, 1

Faherty, J. K., Burgasser, A. J., Walter, F. M., et al. 2012, ApJ, 752, 56

Faherty, J. K., Riedel, A. R., Cruz, K. L., et al. 2016, ApJS, 225, 10

Finch, C. T., \& Zacharias, N. 2016, AJ, 151, 160

Fitzgerald, M. P. 1968, AJ, 73, 983

Freed, M., Close, L. M., \& Siegler, N. 2003, ApJ, 584,453

Frisch, P. C., Redfield, S., \& Slavin, J. D. 2011, ARA\&A, 49, 237 
Fukugita, M., Ichikawa, T., Gunn, J. E., et al. 1996, AJ, 111, 1748

Gagné, J., Lafrenière, D., Doyon, R., Malo, L., \& Artigau, É. 2014, ApJ, 783, 121

Gaia Collaboration, Brown, A. G. A., Vallenari, A., et al. 2016a, A\&A, 595, A2

Gaia Collaboration, Prusti, T., de Bruijne, J. H. J., et al. 2016b, A\&A, 595, A1

Gatewood, G., \& Coban, L. 2009, AJ, 137, 402

Gatewood, G. D. 1987, AJ, 94, 213

Gawroński, M. P., Goździewski, K., \& Katarzyński, K. 2017, MNRAS, 466, 4211

Giclas, H. L., Burnham, R., \& Thomas, N. G. 1971, Lowell proper motion survey Northern Hemisphere. The G numbered stars. 8991 stars fainter than magnitude 8 with motions $>0.26^{\prime \prime} /$ year

Giclas, H. L., Burnham, Jr., R., \& Thomas, N. G. 1978, Lowell Observatory Bulletin, 8, 89

Gizis, J., \& Reid, I. 1997a, PASP, 109, 1232

Gizis, J. E. 1997, AJ, 113, 806

Gizis, J. E., \& Harvin, J. 2006, AJ, 132, 2372

Gizis, J. E., \& Reid, I. N. 1997b, PASP, 109, 849

Green, P. 2013, ApJ, 765, 12

Han, I. 1989, AJ, 97, 607

Harris, H. C., Dahn, C. C., \& Dupuy, T. J. 2015, in Cambridge Workshop on Cool Stars, Stellar Systems, and the Sun, Vol. 18, 18th Cambridge Workshop on Cool Stars, Stellar Systems, and the Sun, ed. G. T. van Belle \& H. C. Harris, 413-418

Harris, H. C., Dahn, C. C., \& Monet, D. G. 1997, in ESA Special Publication, Vol. 402, Hipparcos - Venice '97, ed. R. M. Bonnet, E. Høg, P. L. Bernacca, L. Emiliani, A. Blaauw, C. Turon, J. Kovalevsky, L. Lindegren, H. Hassan, M. Bouffard, B. Strim, D. Heger, M. A. C. Perryman, \& L. Woltjer, 105-108

Harris, H. C., Vrba, F. J., Dahn, C. C., et al. 1999, AJ, 117, 339

Harris, H. C., Dahn, C. C., Zacharias, N., et al. 2016, AJ, 152, 118

Hartwick, F. D. A., Cowley, A. P., \& Mould, J. R. 1984, ApJ, 286, 269

Henry, T. J., Jao, W.-C., Subasavage, J. P., et al. 2006, AJ, 132, 2360

Ivezić, Ž., Smith, J. A., Miknaitis, G., et al. 2007, AJ, 134, 973

Ivezic, Z., Axelrod, T., Brandt, W. N., et al. 2008, Serbian Astronomical Journal, 176, 1
Jao, W.-C., Henry, T. J., Beaulieu, T. D., \& Subasavage, J. P. 2008, AJ, 136, 840

Jao, W.-C., Henry, T. J., Subasavage, J. P., et al. 2005, AJ, 129, 1954

—. 2011, AJ, 141, 117

Jordi, C., Gebran, M., Carrasco, J. M., et al. 2010, A\&A, 523, A48

Just, A., Fuchs, B., Jahreiß, H., et al. 2015, MNRAS, 451, 149

Khovritchev, M. Y., Izmailov, I. S., \& Khrutskaya, E. V. 2013, MNRAS, 435, 1083

Kirkpatrick, J. D., Henry, T. J., \& McCarthy, Jr., D. W. 1991, ApJS, 77, 417

Kirkpatrick, J. D., Reid, I. N., Liebert, J., et al. 1999, ApJ, 519, 802

Kirkpatrick, J. D., Looper, D. L., Burgasser, A. J., et al. 2010, ApJS, 190, 100

Kirkpatrick, J. D., Kellogg, K., Schneider, A. C., et al. 2016, ApJS, 224, 36

Koerner, D. W., Kirkpatrick, J. D., McElwain, M. W., \& Bonaventura, N. R. 1999, ApJL, 526, L25

Konopacky, Q. M., Ghez, A. M., Barman, T. S., et al. 2010, ApJ, 711, 1087

Koren, S. C., Blake, C. H., Dahn, C. C., \& Harris, H. C. 2016, AJ, 151, 57

Landolt, A. U. 1983, AJ, 88, 439

—. 1992, AJ, 104, 340

—. 2007, AJ, 133, 2502

—. 2013, AJ, 146, 131

Leggett, S. K., \& Hawkins, M. R. S. 1989, MNRAS, 238, 145

Lépine, S., Rich, R. M., \& Shara, M. M. 2003, AJ, 125, 1598

—. 2007, ApJ, 669, 1235

Lépine, S., \& Scholz, R.-D. 2008, ApJL, 681, L33

Lépine, S., Shara, M. M., \& Rich, R. M. 2002, AJ, 124,1190

—. 2004, ApJL, 602, L125

Levinson, F. H., \& Ianna, P. A. 1977, AJ, 82, 299

Liebert, J., Dahn, C. C., Gresham, M., \& Strittmatter, P. A. 1979, ApJ, 233, 226

Lindegren, L. 1980, A\&A, 89, 41

Lindegren, L., Lammers, U., Bastian, U., et al. 2016, A\&A, 595, A4

Liu, M. C., Dupuy, T. J., \& Allers, K. N. 2016, ApJ, 833, 96

Liu, M. C., \& Leggett, S. K. 2005, ApJ, 634, 616

Lodieu, N., Burgasser, A. J., Pavlenko, Y., \& Rebolo, R. 2015, A\&A, 579, A58 
Lodieu, N., Espinoza Contreras, M., Zapatero

Osorio, M. R., et al. 2017, A\&A, 598, A92

Luhman, K. L., Loutrel, N. P., McCurdy, N. S., et al. 2012, ApJ, 760, 152

Lutz, T. E., \& Upgren, A. R. 1980, AJ, 85, 1390

Luyten, W. J. 1964, Proper Motion Survey with the Forty-Eight Inch Schmidt Telescope.

(Minneapolis, MN: University of Minnesota)

—. 1979, LHS Catalogue. Second edition.

(Minneapolis, MN: University of Minnesota)

—. 1980, NLTT Catalogue. Volume_III. 0_-to -30_. (Minneapolis, MN: University of Minnesota)

Luyten, W. J., \& Hughes, H. S. 1983, Proper Motion Survey, University of Minnesota, 63

MacConnell, D. J., Osborn, W. H., \& Miller, R. J. 1997, AJ, 114, 1268

Monet, D. G., \& Dahn, C. C. 1983, AJ, 88, 1489

Monet, D. G., Dahn, C. C., Vrba, F. J., et al. 1992, AJ, 103, 638

Pravdo, S. H., \& Shaklan, S. B. 2009, ApJ, 700, 623

Reid, I. N., Gizis, J. E., Kirkpatrick, J. D., \& Koerner, D. W. 2001, AJ, 121, 489

Reid, I. N., \& Hawley, S. L. 1999, AJ, 117, 343

Reid, I. N., Lewitus, E., Allen, P. R., Cruz, K. L., \& Burgasser, A. J. 2006a, AJ, 132, 891

Reid, I. N., Lewitus, E., Burgasser, A. J., \& Cruz, K. L. 2006b, ApJ, 639, 1114

Reid, I. N., Kirkpatrick, J. D., Liebert, J., et al. 1999, ApJ, 521, 613

Riedel, A. R., Subasavage, J. P., Finch, C. T., et al. 2010, AJ, 140, 897

Sahlmann, J., Lazorenko, P. F., Ségransan, D., et al. 2014, A\&A, 565, A20

Salim, S., \& Gould, A. 2003, ApJ, 582, 1011

Schilbach, E., Röser, S., \& Scholz, R.-D. 2009, A\&A, 493, L27

Schlieder, J. E., Lépine, S., \& Simon, M. 2010, AJ, 140, 119

—. 2012, AJ, 143, 80

Scholz, R.-D., Lehmann, I., Matute, I., \&

Zinnecker, H. 2004, A\&A, 425, 519
Schweitzer, A., Scholz, R.-D., Stauffer, J., Irwin, M., \& McCaughrean, M. J. 1999, A\&A, 350, L62

Skrutskie, M. F., Cutri, R. M., Stiening, R., et al. 2006, AJ, 131, 1163

Smart, R. L., Ioannidis, G., Jones, H. R. A., Bucciarelli, B., \& Lattanzi, M. G. 2010, A\&A, 514, A84

Smart, R. L., Lattanzi, M. G., Jahreiß, H., Bucciarelli, B., \& Massone, G. 2007, A\&A, 464, 787

Smith, J. A., Tucker, D. L., Kent, S., et al. 2002, AJ, 123, 2121

Stetson, P. B. 1987, PASP, 99, 191

Strand, K. A. 1964, S\&T, 27

Teegarden, B. J., Pravdo, S. H., Hicks, M., et al. 2003, ApJL, 589, L51

Tinney, C. G. 1996, MNRAS, 281, 644

Tinney, C. G., Reid, I. N., Gizis, J., \& Mould, J. R. 1995, AJ, 110, 3014

van Altena, W. F., Lee, J. T., \& Hoffleit, E. D. 1995, The General Catalogue of Trigonometric Stellar Parallaxes (4th edition; New Haven, CT: Yale University Observatory)

Vrba, F. J., Henden, A. A., Luginbuhl, C. B., et al. 2004, AJ, 127, 2948

Wang, Y., Jones, H. R. A., Smart, R. L., et al. 2014, PASP, 126, 15

Weinberger, A. J., Boss, A. P., Keiser, S. A., et al. 2016, AJ, 152, 24

Woolf, V. M., Lépine, S., \& Wallerstein, G. 2009, PASP, 121, 117

Zapatero Osorio, M. R., Béjar, V. J. S., Miles-Páez, P. A., et al. 2014, A\&A, 568, A6

Zhang, Z. H., Homeier, D., Pinfield, D. J., et al. 2017a, MNRAS, 468, 261

Zhang, Z. H., Pinfield, D. J., Gálvez-Ortiz, M. C., et al. 2017b, MNRAS, 464, 3040 
Facility: USNO:61in (TEK2K, EEV24, TI800), USNO:40in

Software: DAOPHOT (Stetson 1987) 